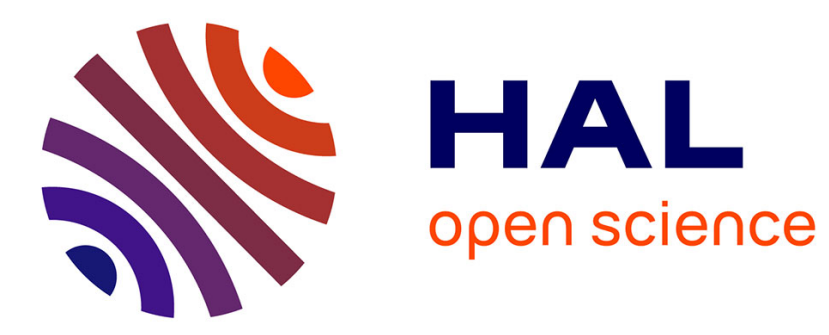

\title{
3D unsteady Navier-Stokes problem with memory and subdifferential boundary condition
}

Mahdi Boukrouche, Laetitia Paoli

\section{To cite this version:}

Mahdi Boukrouche, Laetitia Paoli. 3D unsteady Navier-Stokes problem with memory and subdifferential boundary condition. Journal of Mathematical Analysis and Applications, 2018, 10.1016/j.jmaa.2018.06.035 . hal-02077741

\section{HAL Id: hal-02077741 \\ https://hal.science/hal-02077741}

Submitted on 12 Apr 2019

HAL is a multi-disciplinary open access archive for the deposit and dissemination of scientific research documents, whether they are published or not. The documents may come from teaching and research institutions in France or abroad, or from public or private research centers.
L'archive ouverte pluridisciplinaire HAL, est destinée au dépôt et à la diffusion de documents scientifiques de niveau recherche, publiés ou non, émanant des établissements d'enseignement et de recherche français ou étrangers, des laboratoires publics ou privés. 


\title{
3D unsteady Navier-Stokes problem with memory and subdifferential boundary condition
}

\author{
Mahdi Boukrouche ${ }^{1}$, Laetitia Paoli ${ }^{1, *}$ \\ Lyon University, UJM F-42023 Saint-Etienne, CNRS UMR 5208, Institut Camille Jordan, \\ 23 rue Paul Michelon, 42023 Saint-Etienne Cedex 2, France
}

\begin{abstract}
We consider a mathematical model which describes the motion of a 3D unsteady fluid flow governed by the Navier-Stokes system, and subjected to mixed boundary conditions with a given velocity on one part of the boundary and nonlinear slip conditions with a memory term reminiscent of Coulomb's friction law on the other part. We establish first some regularity properties and estimates for a simplified model. Then we prove the existence of a solution to our problem by using a successive approximation technique and compactness arguments based on Helly's theorem for the velocity field.
\end{abstract}

Keywords: Navier-Stokes system, Coulomb's friction law, history-dependent boundary condition, variational inequality, successive approximation technique.

\section{Introduction}

Motivated by lubrication or injection/extrusion problems, we consider in this paper a 3D incompressible fluid flow with mixed boundary conditions, namely non homogeneous Dirichlet boundary conditions on one part of the boundary and nonlinear slip conditions of friction type on the other part. More precisely let us denote by $\Omega \subset \mathbb{R}^{d}, d=3$, the fluid domain given by

$$
\Omega=\left\{\left(x^{\prime}, x_{3}\right) \in \mathbb{R}^{2} \times \mathbb{R}: \quad x^{\prime} \in \omega, \quad 0<x_{3}<h\left(x^{\prime}\right)\right\}
$$

where $\omega$ is a non empty open bounded subset of $\mathbb{R}^{2}$ with a $C^{1}$ boundary, and $h$ is a function of class $C^{1}$ on $\mathbb{R}^{2}$, bounded from above and from below by two positive real numbers. Let us emphasize that we do not introduce any restrictive assumption on the thickness of the domain unlike previous papers where only thin films where studied $([24,1,6])$. Then the velocity and the pressure fields, $u$ and $p$, are solutions of Navier-Stokes system

$$
\frac{\partial u}{\partial t}+(u \cdot \nabla) u-\operatorname{div}(\sigma)=f \quad \text { in } \quad \Omega \times(0, T),
$$

\footnotetext{
*Corresponding author. Phone: +33477 485112

URL: mahdi.boukrouche@univ-st-etienne.fr (Mahdi Boukrouche), laetitia.paoli@univ-st-etienne.fr (Laetitia Paoli) 


$$
\operatorname{div}(u)=0 \quad \text { in } \Omega \times(0, T),
$$

with $T>0$, and the initial condition

$$
u(0)=u_{0} \quad \text { in } \Omega,
$$

where $f$ is the density of body forces, $\sigma=-p I+2 \mu(\theta) D(u)$ is the stress tensor, $\mu(\theta)$ is the temperature-dependent viscosity of the fluid and $D(u)$ is the strain rate tensor given by

$$
D(u)=\left(d_{i j}(u)\right)_{1 \leq i, j \leq 3}, \quad d_{i j}(u)=\frac{1}{2}\left(\frac{\partial u_{i}}{\partial x_{j}}+\frac{\partial u_{j}}{\partial x_{i}}\right) \quad 1 \leq i, j \leq 3 .
$$

The boundary of the fluid domain is decomposed as $\partial \Omega=\Gamma=\Gamma_{0} \cup \Gamma_{L} \cup \Gamma_{1}$, where $\Gamma_{0}=$ $\left\{\left(x^{\prime}, x_{3}\right) \in \bar{\Omega}: x_{3}=0\right\}$ is the lower surface of the injection/extrusion device, $\Gamma_{1}=\left\{\left(x^{\prime}, x_{3}\right) \in \bar{\Omega}\right.$ : $\left.x_{3}=h\left(x^{\prime}\right)\right\}$ is the upper surface of the device and $\Gamma_{L}=\left\{\left(x^{\prime}, x_{3}\right) \in \bar{\Omega}: 0<x_{3}<h\left(x^{\prime}\right)\right\}$ is the lateral part of the boundary.

To describe the boundary conditions, we denote by $s_{0}: \Gamma_{0} \rightarrow \mathbb{R}^{2}$ the shear velocity of the lower surface at $t=0$ and by $s_{0} \zeta(t)$, its velocity at any instant $t \in[0, T]$ with $\zeta(0)=1$. We denote also by $n=\left(n_{1}, n_{2}, n_{3}\right)$ the unit outward normal vector to $\partial \Omega$, by $u \cdot w$ the Euclidean inner product of two vectors $u$ and $w$, and by $|$.$| the Euclidian norm. We define respectively the normal and the$ tangential velocities on $\partial \Omega$ by

$$
u_{n}=u \cdot n=\sum_{i=1}^{3} u_{i} n_{i}, \quad u_{t}=\left(u_{t i}\right)_{1 \leq i \leq 3} \text { with } u_{t i}=u_{i}-u_{n} n_{i} \quad 1 \leq i \leq 3
$$

and the normal and the tangential components of the stress tensor by

$$
\sigma_{n}=(\sigma \cdot n) \cdot n=\sum_{i, j=1}^{3} \sigma_{i j} n_{i} n_{j}, \quad \sigma_{t}=\left(\sigma_{t i}\right)_{1 \leq i \leq 3} \text { with } \sigma_{t i}=\sum_{j=1}^{3} \sigma_{i j} n_{j}-\sigma_{n} n_{i} \quad 1 \leq i \leq 3 .
$$

We introduce a function $g: \partial \Omega \rightarrow \mathbb{R}^{d}$ such that

$$
\int_{\Gamma_{L}} g_{n} d \gamma=0, \quad g=0 \text { on } \Gamma_{1}, \quad g_{n}=0 \text { and } g_{t}=\left(s_{0}, 0\right) \text { on } \Gamma_{0} .
$$

Such a function exists. Indeed, with the particular geometry of $\Omega$ considered here, the normal at any point $\left(x^{\prime}, x_{3}\right) \in \Gamma_{L}$ is independent of $x_{3}$ and we may consider for instance $g=\left(s_{0}\left(x^{\prime}\right) s_{1}\left(x_{3}\right), 0\right)$ for all $\left(x^{\prime}, x_{3}\right) \in \partial \Omega$ where $s_{1}$ is any $C^{1}$ function on $\mathbb{R}$ such that $s_{1}(0)=1, s_{1}\left(x_{3}\right)=0$ for all $x_{3} \geq h_{m}$ and $\int_{0}^{h_{m}} s_{1}\left(x_{3}\right) d x_{3}=0$ with $h_{m}=\inf _{x^{\prime} \in \mathbb{R}^{2}} h\left(x^{\prime}\right)$.

We assume that the upper surface of the extrusion device is fixed, so

$$
u=0 \quad \text { on } \quad \Gamma_{1} \times(0, T)
$$

and the velocity on the lateral boundary is given by

$$
u=g \zeta \quad \text { on } \quad \Gamma_{L} \times(0, T) .
$$


The normal component of the velocity on the lower part of boundary is given by

$$
u_{n}=0 \quad \text { on } \quad \Gamma_{0} \times(0, T)
$$

while the tangential velocity is unknown and satisfies a friction type boundary condition

$$
\left|\sigma_{t}\right| \leq \mathcal{F} \quad \text { on } \quad \Gamma_{0} \times(0, T)
$$

with

$$
\left.\begin{array}{l}
\left|\sigma_{t}\right|<\mathcal{F} \Rightarrow u_{t}=\left(s_{0} \zeta, 0\right) \\
\left|\sigma_{t}\right|=\mathcal{F} \Rightarrow \exists \lambda \geq 0 \quad \text { such that } \quad u_{t}=\left(s_{0} \zeta, 0\right)-\lambda \sigma_{t}
\end{array}\right\} \quad \Gamma_{0} \times(0, T)
$$

where $\mathcal{F}$ is the upper limit for the shear stress (we say also that $\mathcal{F}$ is the friction threshold), which may be given as a data or which may depend on $\sigma_{n}$. The former case leads to Tresca's friction boundary conditions while the latter is reminiscent of Coulomb's friction boundary conditions for solids $([9,10])$.

The two conditions (7)-(8) can be rewritten as a subdifferential boundary condition

$$
-\sigma_{t} \in \mathcal{F} \partial\left(\left|u_{t}-\left(s_{0} \zeta, 0\right)\right|\right) \quad \text { on } \Gamma_{0} \times(0, T)
$$

where $u_{t}-\left(s_{0} \zeta, 0\right)$ is the relative tangential velocity of the fluid with respect to the lower surface of the injection/extrusion device and $\partial(|\cdot|)$ is the subdifferential of the function $|\cdot|$ (see [25]).

This kind of nonlinear slip boundary conditions for flows have been introduced first for Bingham fluids ([18]) and seems in agreement with experiments ([21, 17]). Then they have been considered also for Stokes and Navier-Stokes flows by H.Fujita during his Lectures at Collège de France ([12]), paving the way to new and challenging mathematical questions. For existence, uniqueness and regularity properties of solutions in the case of Tresca's friction see $[13,14,27,15,28]$ or more recent references [2, 4].

The purpose of this paper is to extend these results to Coulomb's friction type boundary conditions i.e. to consider the case when $\mathcal{F}$ depends on the normal component of the stress tensor. For solids in contact, Coulomb established experimentally that the friction threshold $\mathcal{F}$ is given by $\mathcal{F}=k\left|\sigma_{n}\right|$, where $k:(0, T) \rightarrow \mathbb{R}^{+}$is called the friction coefficient ([7]). Since we expect the velocity field to take its values in $\left(H^{1}(\Omega)\right)^{3}$, we may only expect $\sigma$ to take its values in $\left(L^{2}(\Omega)\right)^{3 \times 3}$ and $\left|\sigma_{n}\right|$ is not well defined on $\Gamma_{0}$. So following $[10,11,8]$ we introduce a regularization operator $\mathcal{R}$ from $H^{-1 / 2}(\partial \Omega)$ to $L^{2}\left(\Gamma_{0}\right)$ by using a convolution technique. Moreover, for this unsteady problem, we have to deal with another difficulty. Indeed the stress tensor has the same regularity with respect to the time variable as the pressure $p$ and we may expect only $\sigma \in H^{-1}\left(0, T ;\left(L^{2}(\Omega)\right)^{3 \times 3}\right)$ except if the friction threshold belongs to $H^{1}\left(0, T ; L^{2}\left(\Gamma_{0}\right)\right)$ (see [2]). Hence we introduce also a time-regularization procedure and we let

$$
\mathcal{F}\left(t, x^{\prime}, \sigma_{n}\right)=k(t) \int_{0}^{t} S(t-s)\left|\mathcal{R}\left(\sigma_{n}(s, \cdot)\right)\left(x^{\prime}\right)\right| d s \quad \forall x^{\prime} \in \Gamma_{0}, \quad \forall t \in[0, T]
$$

where $S$ is a non negative smooth real function which can be interpreted as the kernel of some history-dependent shear stress threshold. Such kind of friction laws have been recently developped in the framework of solid mechanics (see [29] and the references therein for instance). 
Finally having in mind lubrication and injection/extrusion problems where thermal effects can not be neglected, we should introduce also the energy conservation law to describe the evolution of the variable $\theta$. Nevertheless, if the heat capacity and conductivity of the fluid are independent of $u$ and $p$, the heat equation is decoupled from the flow problem and we may consider $\theta$ as a data. More complex behaviours, leading to a coupled system of partial differential equations for the triplet velocity-pressure-temperature have been studied in ([3]) in the case of Tresca's friction boundary conditions.

The paper is organized as follows. In Section 2 we introduce the functional framework and the assumptions on the data, then we formulate the problem as a variational inequality for the fluid velocity and pressure fields. In Section 3 we recall the existence, uniqueness and regularity results for Tresca's problem already obtained in $[2,4]$ and we establish additional regularity properties and estimates for the second time derivative of the velocity in $L^{2}\left(0, T ;\left(\mathbf{H}_{0 d i v}^{1}(\Omega)\right)^{\prime}\right)$ and the pressure in $L^{\infty}\left(0, T ; L_{0}^{2}(\Omega)\right)$. Then in Section 4 we prove the existence of a solution for our problem by using a successive approximation method with respect to the friction threshold and compactness arguments based on Helly's theorem for the velocity field.

\section{Variational formulation of the problem}

From now on let us denote $\mathbf{X}=X^{3}$ for any Banach space $X$. We introduce the following functional framework. Let

$$
\mathcal{V}_{0}=\left\{\varphi \in \mathbf{H}^{1}(\Omega): \varphi=0 \text { on } \Gamma_{L} \cup \Gamma_{1}, \varphi_{n}=0 \text { on } \Gamma_{0}\right\}
$$

and its subspace

$$
\mathcal{V}_{0 d i v}=\left\{\varphi \in \mathcal{V}_{0}: \operatorname{div}(\varphi)=0 \text { in } \Omega\right\},
$$

endowed with the norm of $\mathbf{H}^{1}(\Omega)$, and

$$
L_{0}^{2}(\Omega)=\left\{q \in L^{2}(\Omega): \int_{\Omega} q d x=0\right\} .
$$

Let $\mu \in C^{1}(\mathbb{R} ; \mathbb{R})$ and assume that there exist three real numbers $\mu^{*}, \mu_{*}$ and $\mu_{*}^{\prime}$ such that

$$
0<\mu^{*} \leq 2 \mu(X) \leq \mu_{*}, \quad\left|\mu^{\prime}(X)\right| \leq \mu_{*}^{\prime} \quad \forall X \in \mathbb{R} .
$$

For a given temperature field $\theta \in L^{2}\left(0, T ; L^{2}(\Omega)\right)$, with $T>0$, we define

$$
\begin{aligned}
& a(\theta ; u, v)=\int_{\Omega} 2 \mu(\theta) D(u): D(v) d x=\int_{\Omega} \sum_{i, j=1}^{3} 2 \mu(\theta) d_{i j}(u) d_{i j}(v) d x \\
& \forall(u, v) \in \mathbf{H}^{1}(\Omega) \times \mathbf{H}^{1}(\Omega), \quad \forall \text { a.e. } t \in(0, T),
\end{aligned}
$$

and

$$
b(u, v, w)=\int_{\Omega} \sum_{i, j=1}^{3} u_{i} \frac{\partial v_{j}}{\partial x_{i}} w_{j} d x \quad \forall(u, v, w) \in \mathbf{H}^{1}(\Omega) \times \mathbf{H}^{1}(\Omega) \times \mathbf{H}^{1}(\Omega) .
$$


By definition of $\mathcal{V}_{0}$ we have the identity

$$
b(u, v, w)=-b(u, w, v)-\int_{\Omega} \operatorname{div}(u) v \cdot w d x \quad \forall(u, v, w) \in \mathcal{V}_{0} \times \mathcal{V}_{0} \times \mathcal{V}_{0},
$$

and using Korn's inequality [19], there exists $\alpha>0$ such that, for almost every $t \in(0, T)$, we have

$$
\alpha\|u\|_{\mathbf{H}^{1}(\Omega)}^{2} \leq \int_{\Omega} 2 \mu(\theta) D(u): D(u) d x \leq \mu_{*}\|u\|_{\mathbf{H}^{1}(\Omega)}^{2} \quad \forall u \in \mathcal{V}_{0}
$$

We assume also that

$$
\begin{gathered}
k \in W^{1, \infty}\left(0, T ; \mathbb{R}^{+}\right), \quad S \in C^{1}\left(\mathbb{R}^{+} ; \mathbb{R}^{+}\right), \\
f \in H^{1}\left(0, T ; \mathbf{L}^{2}(\Omega)\right), \\
\theta \in W^{1, \infty}\left(0, T ; L^{\infty}(\Omega)\right), \quad \theta(0) \in W^{1,4}(\Omega), \\
\zeta \in C^{\infty}([0, T]) \quad \text { such that } \quad \zeta(0)=1,
\end{gathered}
$$

and

$$
u_{0} \in \mathbf{H}^{2}(\Omega), \quad \operatorname{div}\left(u_{0}\right)=0 \text { in } \Omega, \quad u_{0}=g \text { on } \partial \Omega, \quad \frac{\partial u_{0}}{\partial x_{d}}=0 \text { on } \Gamma_{0}
$$

with $d=3$. We set

$$
v=u-u_{0} \zeta
$$

The choice of the particular boundary condition (5) on $\Gamma_{L}$ plays here a role in the definition of $v$. For the mathematical study this new unknown velocity satisfies two important properties: it vanishes on $\Gamma_{L} \cup \Gamma_{1}$ and at $t=0$. Then the variational formulation of problem (1)-(9) is obtained by multiplying (1) by test-functions and using Green's formula. Of course we expect $v \in L^{2}\left(0, T ; \mathcal{V}_{0 \text { div }}\right)$ but since we consider a 3D flow domain the time derivative $\frac{\partial v}{\partial t}$ is expected to belong only to $L^{4 / 3}\left(0, T ;\left(\mathcal{V}_{0 \text { div }}\right)^{\prime}\right)$ (see [4]). So we choose test-functions as $\varphi \chi \in \mathcal{V}_{0} \times \mathcal{D}(0, T)$. It follows that $(v, \varphi) \in L^{2}(0, T ; \mathbb{R})$ where $(\cdot, \cdot)$ denotes the inner product in $\mathbf{L}^{2}(\Omega)$, and $\frac{\partial}{\partial t}(v, \varphi)$ is defined in the sense of distributional derivatives. Moreover Tresca's boundary condition (7)-(8) is equivalent to

$$
\left.\begin{array}{l}
\left|\sigma_{t}\right| \leq \mathcal{F} \\
v_{t} \cdot \sigma_{t}+\mathcal{F}\left|v_{t}\right|=0
\end{array}\right\} \quad \text { on } \Gamma_{0} \times(0, T)
$$

(see for instance [9]) and the boundary term derived from Green's formula can be rewritten as

$$
-\int_{0}^{T} \int_{\Gamma_{0}} \sigma_{t} \varphi \chi d x^{\prime} d t=-A+\int_{0}^{T} \int_{\Gamma_{0}} \mathcal{F}(|v+\varphi \chi|-|v|) d x^{\prime} d t
$$




$$
\begin{aligned}
A & =\int_{0}^{T} \int_{\Gamma_{0}} \sigma_{t}(\varphi \chi+v) d x^{\prime} d t+\int_{0}^{T} \int_{\Gamma_{0}} \mathcal{F}|v+\varphi \chi| d x^{\prime} d t \\
& \geq \int_{0}^{T} \int_{\Gamma_{0}} \underbrace{\left(-\left|\sigma_{t}\right|+\mathcal{F}\right)}_{\geq 0}|v+\varphi \chi| d x^{\prime} d t \geq 0
\end{aligned}
$$

So we obtain

Problem (P) For $k, f, \theta, \zeta$ and $v_{0}$ such that (13)-(17) hold, find

$$
v \in L^{2}\left(0, T ; \mathcal{V}_{0 \text { div }}\right) \cap L^{\infty}\left(0, T ; \mathbf{L}^{2}(\Omega)\right) \quad \text { such that } \frac{\partial v}{\partial t} \in L^{4 / 3}\left(0, T ;\left(\mathcal{V}_{0 \text { div }}\right)^{\prime}\right)
$$

and

$$
p \in H^{-1}\left(0, T ; L_{0}^{2}(\Omega)\right)
$$

such that $\mathcal{F}\left(\cdot, \cdot, \sigma_{n}\right) \in L^{2}\left(0, T ; L^{2}\left(\Gamma_{0}\right)\right)$ and satisfying the following variational inequality

$$
\begin{aligned}
& \left\langle\frac{\partial}{\partial t}(v, \varphi), \chi\right\rangle+\langle b(v, v, \varphi), \chi\rangle-\langle(p, \operatorname{div}(\varphi)), \chi\rangle+\langle a(\theta ; v, \varphi), \chi\rangle \\
& +\Psi(v+\varphi \chi)-\Psi(v) \geq\langle(f, \varphi), \chi\rangle-\left\langle\zeta a\left(\theta ; u_{0}, \varphi\right), \chi\right\rangle-\left\langle\zeta^{\prime}\left(u_{0}, \varphi\right), \chi\right\rangle \\
& -\left\langle\zeta b\left(u_{0}, v+u_{0} \zeta, \varphi\right), \chi\right\rangle-\left\langle\zeta b\left(v, u_{0}, \varphi\right), \chi\right\rangle \quad \forall(\varphi, \chi) \in \mathcal{V}_{0} \times \mathcal{D}(0, T),
\end{aligned}
$$

with

$$
\begin{aligned}
\Psi(v) & =\int_{0}^{T} \int_{\Gamma_{0}} \mathcal{F}\left(t, x^{\prime}, \sigma_{n}\right)\left|v\left(t, x^{\prime}\right)\right| d x^{\prime} d t \\
& =\int_{0}^{T} k(t) \int_{\Gamma_{0}}\left(\int_{0}^{t} S(t-s)\left|\mathcal{R}\left(\sigma_{n}(s, \cdot)\right)\left(x^{\prime}\right)\right| d s\right)\left|v\left(t, x^{\prime}\right)\right| d x^{\prime} d t,
\end{aligned}
$$

and the initial condition

$$
v(0, \cdot)=0
$$

where $\langle\cdot, \cdot\rangle=\langle\cdot, \cdot\rangle_{\mathcal{D}^{\prime}(0, T), \mathcal{D}(0, T)}$ is the duality product between $\mathcal{D}^{\prime}(0, T)$ and $\mathcal{D}(0, T)$. Hence the first term in (19) has to be understood as the derivative in the distribution sense of $(v, \varphi)$ (which belongs to $L^{2}(0, T ; \mathbb{R})$ for any $\left.\varphi \in \mathcal{V}_{0}\right)$, i.e.

$$
\left\langle\frac{\partial}{\partial t}(v, \varphi), \chi\right\rangle=-\left\langle(v, \varphi), \chi^{\prime}\right\rangle=-\int_{0}^{T}(v(t, \cdot), \varphi) \chi^{\prime}(t) d t \quad \forall(\varphi, \chi) \in \mathcal{V}_{0} \times \mathcal{D}(0, T) .
$$

Let us emphasize that we identify $v+\varphi \chi$ and $v$ with their trace on $\Gamma_{0}$ in the definition of $\Psi(v+\varphi \chi)$ and $\Psi(v)$.

When $\mathcal{F}\left(\cdot, \cdot, \sigma_{n}\right)$ is replaced by a given shear stress threshold $\ell \in L^{2}\left(0, T ; L_{+}^{2}\left(\Gamma_{0}\right)\right)$ in the friction functional, i.e. when $\Psi$ in (19)-(20) is replaced by the functional $\Phi$ given by

$$
\Phi(w)=\int_{0}^{T} \int_{\Gamma_{0}} \ell|w| d x^{\prime} d t \quad \forall w \in L^{2}\left(0, T ; \mathbf{L}^{2}\left(\Gamma_{0}\right)\right)
$$

we get a description of Tresca's friction law at the boundary. For this Tresca's problem the existence of a solution is established in [4] and uniqueness and regularity properties in [2].

Starting from these results we will prove the existence of a strong solution to Coulomb's problem (P) by applying a successive approximation technique with respect to the friction threshold. As a first step we establish in the next section some additional regularity properties for the solution to Tresca's problem. 


\section{Preliminary results}

Let us recall first the existence, uniqueness and regularity results for Tresca's problem.

Theorem 3.1 (Existence and uniqueness for Tresca's problem). [2, 4] Assume that (10), (16), (17) hold and $f \in L^{2}\left(0, T ; \mathbf{L}^{2}(\Omega)\right), \theta \in L^{2}\left(0, T ; L^{2}(\Omega)\right), \ell \in L^{2}\left(0, T ; L_{+}^{2}\left(\Gamma_{0}\right)\right)$. Then there exists (at least) one solution $(v, p)$ to Tresca's problem i.e. there exists

$$
v \in L^{2}\left(0, T ; \mathcal{V}_{0 \text { div }}\right) \cap L^{\infty}\left(0, T ; \mathbf{L}^{2}(\Omega)\right) \text { such that } \frac{\partial v}{\partial t} \in L^{4 / 3}\left(0, T ;\left(\mathcal{V}_{0 d i v}\right)^{\prime}\right)
$$

and

$$
p \in H^{-1}\left(0, T ; L_{0}^{2}(\Omega)\right)
$$

such that

$$
\begin{aligned}
& \left\langle\frac{\partial}{\partial t}(v, \varphi), \chi\right\rangle+\langle b(v, v, \varphi), \chi\rangle-\langle(p, \operatorname{div}(\varphi)), \chi\rangle+\langle a(\theta ; v, \varphi), \chi\rangle \\
& +\Phi(v+\varphi \chi)-\Phi(v) \geq\langle(f, \varphi), \chi\rangle-\left\langle\zeta a\left(\theta ; u_{0}, \varphi\right), \chi\right\rangle-\left\langle\zeta^{\prime}\left(u_{0}, \varphi\right), \chi\right\rangle \\
& -\left\langle\zeta b\left(u_{0}, v+u_{0} \zeta, \varphi\right), \chi\right\rangle-\left\langle\zeta b\left(v, u_{0}, \varphi\right), \chi\right\rangle \quad \forall(\varphi, \chi) \in \mathcal{V}_{0} \times \mathcal{D}(0, T)
\end{aligned}
$$

with

$$
\Phi(v)=\int_{0}^{T} \int_{\Gamma_{0}} \ell\left(t, x^{\prime}\right)\left|v\left(t, x^{\prime}\right)\right| d x^{\prime} d t
$$

and the initial condition

$$
v(0, \cdot)=0
$$

Furthermore Tresca's problem (22)-(24) admits at most one solution ( $v, p)$ such that

$$
v \in L^{8}\left(0, T ; \mathbf{L}^{4}(\Omega)\right) .
$$

Assume now that (14)-(15) hold and $\ell \in H^{1}\left(0, T ; L_{+}^{2}\left(\Gamma_{0}\right)\right)$. Let

$$
\mathcal{D}=\mathcal{D}_{0}+\mathbf{A}_{\mathbf{4}} \exp \left(2 \mathbf{A}_{3} T\right)
$$

where

$$
\begin{aligned}
\mathcal{D}_{0}= & 2\|f(0)\|_{\mathbf{L}^{2}(\Omega)}^{2}+2 T\left\|\frac{\partial f}{\partial t}\right\|_{L^{2}\left(0, T ; \mathbf{L}^{2}(\Omega)\right)}^{2}+\frac{2 \mu_{*}^{2}}{\alpha}\left\|u_{0}\right\|_{\mathbf{H}^{1}(\Omega)}^{2}\|\zeta\|_{C([0, T])}^{2} \\
& +\left\|u_{0}\right\|_{\mathbf{L}^{2}(\Omega)}^{2}\left\|\zeta^{\prime}\right\|_{C([0, T])}^{2}+K^{4}\left\|u_{0}\right\|_{\mathbf{H}^{1}(\Omega)}^{2}\left\|u_{0}\right\|_{\mathbf{H}^{2}(\Omega)}^{2}\|\zeta\|_{C([0, T])}^{4} \\
& +\left(4+\frac{2 K^{4}}{\alpha}\left\|u_{0}\right\|_{\mathbf{H}^{2}(\Omega)}^{2}\|\zeta\|_{C([0, T])}^{2} \|\right) C_{1}^{2} \\
C_{1}^{2}= & \max \left(1, \frac{1}{\alpha}\right) C_{1}^{\prime} \exp \left(C_{1}^{\prime \prime} T\right)
\end{aligned}
$$




$$
\begin{aligned}
C_{1}^{\prime}= & \|f\|_{L^{2}\left(0, T ; \mathbf{L}^{2}(\Omega)\right)}^{2}+\frac{2 \mu_{*}^{2}}{\alpha}\left\|u_{0}\right\|_{\mathbf{H}^{1}(\Omega)}^{2}\|\zeta\|_{L^{2}(0, T)}^{2}+\left\|u_{0}\right\|_{\mathbf{L}^{2}(\Omega)}^{2}\left\|\zeta^{\prime}\right\|_{L^{2}(0, T)}^{2} \\
& +K^{4}\left\|u_{0}\right\|_{\mathbf{H}^{1}(\Omega)}^{2}\left\|\nabla u_{0}\right\|_{\mathbf{H}^{1}(\Omega)}^{2}\|\zeta\|_{L^{4}(0, T)}^{4} \\
C_{1}^{\prime \prime}= & 3+\frac{2 K^{4}}{\alpha}\left\|\nabla u_{0}\right\|_{\mathbf{H}^{1}(\Omega)}^{2}\|\zeta\|_{L^{\infty}(0, T)}^{2} \\
\mathbf{A}_{\mathbf{3}}= & 1+\frac{7 K^{4}}{2 \alpha}\left\|u_{0}\right\|_{\mathbf{H}^{2}(\Omega)}^{2}\|\zeta\|_{C([0, T])}^{2} \\
\mathbf{A}_{\mathbf{4}}^{\prime}= & \mathbf{A}_{\mathbf{0}}^{\mathbf{2}}+2\left\|\frac{\partial f}{\partial t}\right\|_{L^{2}\left(0, T ; \mathbf{L}^{2}(\Omega)\right)}^{2}+2 \mathbf{A}_{\mathbf{1}} T+2 \mathbf{A}_{2} C_{1}^{2} \\
\mathbf{A}_{\mathbf{4}}= & \mathbf{A}_{\mathbf{4}}^{\prime}+\frac{7 \gamma(\Omega)^{2}}{\alpha}\left\|\frac{\partial \ell}{\partial t}\right\|_{L^{2}\left(0, T ; L^{2}\left(\Gamma_{0}\right)\right)}^{2}
\end{aligned}
$$

where $K$ is the norm of the identity mapping from $\mathbf{H}^{1}(\Omega)$ into $\mathbf{L}^{4}(\Omega), \gamma(\Omega)$ is the norm of the trace operator from $\mathbf{H}^{1}(\Omega)$ into $\mathbf{L}^{2}(\partial \Omega)$ and

$$
\begin{aligned}
\mathbf{A}_{\mathbf{0}}= & K^{2}\left\|u_{0}\right\|_{\mathbf{H}^{1}(\Omega)}\left\|u_{0}\right\|_{\mathbf{H}^{2}(\Omega)}+2 K \mu_{*}^{\prime}\|\nabla(\theta(0))\|_{\mathbf{L}^{4}(\Omega)}\left\|u_{0}\right\|_{\mathbf{H}^{1}(\Omega)} \\
& +\mu_{*}\left\|u_{0}\right\|_{\mathbf{H}^{2}(\Omega)}+\|f(0)\|_{\mathbf{L}^{2}(\Omega)}+\left\|u_{0}\right\|_{L^{2}(\Omega)}\left|\zeta^{\prime}(0)\right| \\
\mathbf{A}_{\mathbf{1}}= & \frac{7 \mu_{*}^{2}}{2 \alpha}\left\|u_{0}\right\|_{\mathbf{H}^{1}(\Omega)}^{2}\left\|\zeta^{\prime}\right\|_{C([0, T])}^{2}+\frac{14\left(\mu_{*}^{\prime}\right)^{2}}{\alpha}\left\|\frac{\partial \theta}{\partial t}\right\|_{L^{\infty}\left(0, T ; L^{\infty}(\Omega)\right)}^{2}\left\|u_{0}\right\|_{\mathbf{H}^{1}(\Omega)}^{2}\|\zeta\|_{C([0, T])}^{2} \\
& +\left\|u_{0}\right\|_{\mathbf{L}^{2}(\Omega)}^{2}\left\|\zeta^{\prime \prime}\right\|_{C([0, T])}^{2}+4 K^{4}\left\|u_{0}\right\|_{\mathbf{H}^{1}(\Omega)}^{2}\left\|u_{0}\right\|_{\mathbf{H}^{2}(\Omega)}^{2}\|\zeta\|_{C([0, T])}^{2}\left\|\zeta^{\prime}\right\|_{C([0, T])}^{2}, \\
\mathbf{A}_{2}= & \frac{14\left(\mu_{*}^{\prime}\right)^{2}}{\alpha}\left\|\frac{\partial \theta}{\partial t}\right\|_{L^{\infty}\left(0, T ; L^{\infty}(\Omega)\right)}^{2}+\frac{7 K^{4}}{2 \alpha}\left\|u_{0}\right\|_{\mathbf{H}^{1}(\Omega)}^{2}\left\|\zeta^{\prime}\right\|_{C([0, T])}^{2} \\
& +K^{4}\left\|u_{0}\right\|_{\mathbf{H}^{2}(\Omega)}^{2}\left\|\zeta^{\prime}\right\|_{C([0, T])}^{2} .
\end{aligned}
$$

Then we have the following regularity results:

Theorem 3.2 (Regularity for Tresca's problem). [2] Assume (10), (14)-(17) and $\ell \in H^{1}\left(0, T ; L_{+}^{2}\left(\Gamma_{0}\right)\right)$. Assume furthermore that

$$
\mathcal{D}<\frac{\alpha^{3}}{9 K^{4}} .
$$

Then Tresca's problem admits (at least) one solution $(v, p)$ such that

$$
\frac{\partial v}{\partial t} \in L^{2}\left(0, T ; \mathcal{V}_{0 d i v}\right) \cap L^{\infty}\left(0, T ; \mathbf{L}^{2}(\Omega)\right), \quad p \in L^{2}\left(0, T ; L_{0}^{2}(\Omega)\right) .
$$

Moreover

$$
\begin{gathered}
\|v(s)\|_{\mathbf{L}^{2}(\Omega)}^{2}+\alpha \int_{0}^{s}\|v\|_{\mathbf{H}^{1}(\Omega)}^{2} d s \leq C_{1}^{\prime} \exp \left(C_{1}^{\prime \prime} s\right) \quad \forall \text { a.e } s \in[0, T], \\
\left\|\frac{\partial v}{\partial t}(s)\right\|_{\mathbf{L}^{2}(\Omega)}^{2}+\left(\alpha-3 K^{2} \sqrt{\frac{\mathcal{D}}{\alpha}}\right) \int_{0}^{s}\left\|\frac{\partial v}{\partial t}\right\|_{\mathbf{H}^{1}(\Omega)}^{2} d t \leq \mathbf{A}_{4} \exp \left(2 \mathbf{A}_{\mathbf{3}} s\right) \quad \forall \text { a.e. } s \in[0, T],
\end{gathered}
$$


and

$$
\|v\|_{L^{\infty}\left(0, T ; \mathbf{H}^{1}(\Omega)\right)} \leq \sqrt{\frac{\mathcal{D}}{\alpha}}<\frac{\alpha}{3 K^{2}}
$$

Remark 3.1. Since

$$
H^{1}\left(0, T ; \mathcal{V}_{0 \text { div }}\right) \subset L^{\infty}\left(0, T ; \mathbf{H}^{1}(\Omega)\right) \subset L^{8}\left(0, T ; \mathbf{L}^{4}(\Omega)\right)
$$

we infer from Theorem 3.1 that such a solution is unique. In the rest of the paper we will call this solution the unique regular solution of Tresca's problem.

All these results allow us to establish now an additional property which will play a crucial role in the proof of our main Theorem 4.4 in the next section.

Proposition 3.3. Let us assume that $\ell \in H^{1}\left(0, T ; L_{+}^{2}\left(\Gamma_{0}\right)\right)$, (10), (14)-(17) hold and condition (26) is satisfied. The unique regular solution $(v, p)$ of Tresca's problem obtained at Theorem 3.2 satisfies also

$$
\frac{\partial^{2} v}{\partial t^{2}} \in L^{2}\left(0, T ;\left(\mathbf{H}_{0 d i v}^{1}(\Omega)\right)^{\prime}\right) \quad \text { and } \quad p \in L^{\infty}\left(0, T ; L_{0}^{2}(\Omega)\right)
$$

with $\mathbf{H}_{0 \operatorname{div}}^{1}(\Omega)=\left\{\varphi \in \mathbf{H}_{0}^{1}(\Omega): \operatorname{div}(\varphi)=0\right\}$.

Proof. Indeed we have

$$
\begin{aligned}
& \left\langle\frac{\partial}{\partial t}(v, \varphi), \chi\right\rangle+\langle b(v, v, \varphi), \chi\rangle-\langle(p, \operatorname{div}(\varphi)), \chi\rangle+\langle a(\theta ; v, \varphi), \chi\rangle \\
& +\int_{0}^{T} \int_{\Gamma_{0}} \ell(|v+\varphi \chi|-|v|) d x^{\prime} d t \geq\langle(f, \varphi), \chi\rangle-\left\langle\zeta a\left(\theta ; u_{0}, \varphi\right), \chi\right\rangle-\left\langle\zeta^{\prime}\left(u_{0}, \varphi\right), \chi\right\rangle \\
& -\left\langle\zeta b\left(u_{0}, v+u_{0} \zeta, \varphi\right), \chi\right\rangle-\left\langle\zeta b\left(v, u_{0}, \varphi\right), \chi\right\rangle \quad \forall(\varphi, \chi) \in \mathcal{V}_{0} \times \mathcal{D}(0, T) .
\end{aligned}
$$

By choosing $\varphi \in \mathbf{H}_{0}^{1}(\Omega)$ then taking $-\chi$ instead $\chi \in \mathcal{D}(0, T)$, and recalling that $\frac{\partial v}{\partial t} \in L^{\infty}\left(0, T ; \mathbf{L}^{2}(\Omega)\right.$, we obtain

$$
\begin{aligned}
& \langle(p, \operatorname{div}(\varphi)), \chi\rangle=\left\langle\left(\frac{\partial v}{\partial t}, \varphi\right), \chi\right\rangle+\left\langle b\left(v+u_{0} \zeta, v+u_{0} \zeta, \varphi\right), \chi\right\rangle+\left\langle a\left(\theta ; v+u_{0} \zeta, \varphi\right), \chi\right\rangle \\
& -\langle(f, \varphi), \chi\rangle+\left\langle\zeta^{\prime}\left(u_{0}, \varphi\right), \chi\right\rangle \quad \forall(\varphi, \chi) \in \mathbf{H}_{0}^{1}(\Omega) \times \mathcal{D}(0, T) .
\end{aligned}
$$

Let $\widetilde{w} \in L^{2}(\Omega)$ and $w \in L_{0}^{2}(\Omega)$ be defined as

$$
w=\widetilde{w}-\frac{1}{|\Omega|} \int_{\Omega} \widetilde{w} d x .
$$

There exists a linear operator $P \in \mathcal{L}\left(L_{0}^{2}(\Omega), \mathbf{H}_{0}^{1}(\Omega)\right)$ such that $\operatorname{div}(P(w))=w$ for all $w \in L_{0}^{2}(\Omega)$ (see [20] page 15 when $\partial \Omega$ is smooth and [16] Corollary 2.4 page 25 when $\Omega$ is a connected bounded subset of $\mathbb{R}^{d}$ with a Lipschitz continuous boundary). Hence

$$
\begin{aligned}
& \langle(p, \widetilde{w}), \chi\rangle=\langle(p, w), \chi\rangle=\left\langle\left(\frac{\partial v}{\partial t}, P(w)\right), \chi\right\rangle+\left\langle b\left(v+u_{0} \zeta, v+u_{0} \zeta, P(w)\right), \chi\right\rangle \\
& +\left\langle a\left(\theta ; v+u_{0} \zeta, P(w)\right), \chi\right\rangle-\langle(f, P(w)), \chi\rangle_{9}+\left\langle\zeta^{\prime}\left(u_{0}, P(w)\right), \chi\right\rangle
\end{aligned}
$$


It follows that

$$
\begin{aligned}
& |\langle(p, \widetilde{w}), \chi\rangle| \leq\left\|\frac{\partial v}{\partial t}\right\|_{L^{\infty}\left(0, T ; \mathbf{L}^{2}(\Omega)\right)}\|P(w) \chi\|_{L^{1}\left(0, T ; \mathbf{L}^{2}(\Omega)\right)} \\
& +\left\|v+u_{0} \zeta\right\|_{L^{\infty}\left(0, T ; \mathbf{L}^{4}(\Omega)\right)}\left\|v+u_{0} \zeta\right\|_{L^{\infty}\left(0, T ; \mathbf{H}^{1}(\Omega)\right)}\|P(w) \chi\|_{L^{1}\left(0, T ; \mathbf{L}^{4}(\Omega)\right)} \\
& +\mu_{*}\left\|v+u_{0} \zeta\right\|_{L^{\infty}\left(0, T ; \mathbf{H}^{1}(\Omega)\right)}\|P(w) \chi\|_{L^{1}\left(0, T ; \mathbf{H}^{1}(\Omega)\right)} \\
& +\|f\|_{L^{\infty}\left(0, T ; \mathbf{L}^{2}(\Omega)\right)}\|P(w) \chi\|_{L^{1}\left(0, T ; \mathbf{L}^{2}(\Omega)\right)} \\
& +\left\|\zeta^{\prime}\right\|_{C([0, T])}\left\|u_{0}\right\|_{\mathbf{L}^{2}(\Omega)}\|P(w) \chi\|_{L^{1}\left(0, T ; \mathbf{L}^{2}(\Omega)\right)}
\end{aligned}
$$

Finally, observing that

$$
\begin{aligned}
\|P(w)\|_{\mathbf{H}^{1}(\Omega)} & \leq\|P\|_{\mathcal{L}\left(L_{0}^{2}(\Omega), \mathbf{H}_{0}^{1}(\Omega)\right)}\|w\|_{\mathbf{L}^{2}(\Omega)} \\
& \leq\|P\|_{\mathcal{L}\left(L_{0}^{2}(\Omega), \mathbf{H}_{0}^{1}(\Omega)\right)}\|\widetilde{w}\|_{\mathbf{L}^{2}(\Omega)}
\end{aligned}
$$

we may conclude that $p \in L^{\infty}\left(0, T ; L_{0}^{2}(\Omega)\right)$.

In order to prove that $\frac{\partial^{2} v}{\partial t^{2}}$ belongs to $L^{2}\left(0, T ;\left(\mathbf{H}_{0 d i v}^{1}(\Omega)\right)^{\prime}\right)$, we apply the same kind of arguments as in [5]. Indeed, the unique regular solution of Tresca's problem is obtained as the limit of the solutions of the approximate problems $\left(P_{\varepsilon}^{\delta}\right)$ introduced in [2,4] when the parameters $\delta$ and $\varepsilon$ tend to zero. More precisely, let $\left(w_{i}\right)_{i \geq 1}$ be a Hilbertian basis of $\mathcal{V}_{0}$ which is orthonormal for the inner product of $\mathbf{L}^{2}(\Omega)$, and orthogonal for the inner product of $\mathbf{H}^{1}(\Omega)$ (the existence of such a basis is a direct consequence of Theorem 6.2-1 page 137 in [23]). The approximate problems $\left(P_{\varepsilon}^{\delta}\right)$ are solved by using Galerkin method and for all $\varepsilon>0, \delta>0$ and $m \geq 1$, we have $v_{\varepsilon m}^{\delta}:[0, T] \rightarrow \operatorname{Span}\left(w_{1}, \ldots, w_{m}\right)$ such that

$$
\begin{aligned}
& \left(\frac{\partial v_{\varepsilon m}^{\delta}}{\partial t}, w_{k}\right)+b\left(v_{\varepsilon m}^{\delta}, v_{\varepsilon m}^{\delta}, w_{k}\right)+\frac{1}{2} \int_{\Omega} \operatorname{div}\left(v_{\varepsilon m}^{\delta}\right) v_{\varepsilon m}^{\delta} \cdot w_{k} d x+\frac{1}{\delta}\left(\operatorname{div}\left(v_{\varepsilon m}^{\delta}\right), \operatorname{div}\left(w_{k}\right)\right) \\
& \quad+a\left(\theta ; v_{\varepsilon m}^{\delta}, w_{k}\right)+\int_{\Gamma_{0}} \ell \frac{v_{\varepsilon m}^{\delta} \cdot w_{k}}{\sqrt{\varepsilon^{2}+\left|v_{\varepsilon m}^{\delta}\right|^{2}}} d x^{\prime}=\left(f, w_{k}\right)-\zeta a\left(\theta ; u_{0}, w_{k}\right) \\
& -\zeta^{\prime}\left(u_{0}, w_{k}\right)-\zeta b\left(u_{0}, v_{\varepsilon m}^{\delta}+u_{0} \zeta, w_{k}\right)-\zeta b\left(v_{\varepsilon m}^{\delta}, u_{0}, w_{k}\right) \quad \text { a.e. in }(0, T),
\end{aligned}
$$

for all $k \in\{1, \cdots, m\}$, with

$$
v_{\varepsilon m}^{\delta}(0, \cdot)=0
$$

Under the previous assumptions we have $v_{\varepsilon m}^{\delta} \in H^{2}\left(0, T\right.$; $\left.\operatorname{Span}\left\{w_{1}, \ldots, w_{m}\right\}\right)$ and (32) holds for all $t \in[0, T]$. So we may differentiate (32) with respect to the time variable and we get 


$$
\begin{aligned}
& \left(\frac{\partial^{2} v_{\varepsilon m}^{\delta}}{\partial t^{2}}, w\right)+b\left(\frac{\partial v_{\varepsilon m}^{\delta}}{\partial t}, v_{\varepsilon m}^{\delta}, w\right)+b\left(v_{\varepsilon m}^{\delta}, \frac{\partial v_{\varepsilon m}^{\delta}}{\partial t}, w\right) \\
& +\frac{1}{2} \int_{\Omega} \operatorname{div}\left(\widehat{v}_{\varepsilon m}^{\delta}\right) \frac{\widetilde{v}_{\varepsilon m}^{\delta}}{\partial t} \cdot w d x+\frac{1}{2} \int_{\Omega} \operatorname{div}\left(\frac{\widetilde{v}_{\varepsilon m}^{\delta}}{\partial t}\right) \widetilde{v}_{\varepsilon m}^{\delta} \cdot w d x+\frac{1}{\delta}\left(\operatorname{div}\left(\frac{\widetilde{v}_{\varepsilon m}^{\delta}}{\partial t}\right), \operatorname{div}(w)\right) \\
& +\int_{\Omega} 2 \mu^{\prime}(\theta) \frac{\partial \theta}{\partial t} D\left(v_{\varepsilon m}^{\delta}\right): D(w) d x+\int_{\Omega} 2 \mu(\theta) D\left(\frac{\partial v_{\varepsilon m}^{\delta}}{\partial t}\right): D(w) d x \\
& +\int_{\Gamma_{0}} \frac{\partial \ell}{\partial t} \frac{v_{\varepsilon m}^{\delta} \cdot w}{\sqrt{\varepsilon^{2}+\left|v_{\varepsilon m}^{\delta}\right|^{2}}} d x^{\prime} \\
& \left.+\int_{\Gamma_{0}} \ell \frac{\frac{\partial v_{\varepsilon m}^{\delta}}{\partial t} \cdot w}{\sqrt{\varepsilon^{2}+\left|v_{\varepsilon m}^{\delta}\right|^{2}}}-\frac{\left(v_{\varepsilon m}^{\delta} \cdot \frac{\partial v_{\varepsilon m}^{\delta}}{\partial t}\right)\left(v_{\varepsilon m}^{\delta} \cdot w\right)}{\left.\left(\varepsilon^{2}+\left|v_{\varepsilon m}^{\delta}\right|^{2}\right)^{3 / 2}\right)}\right) d x^{\prime} \\
& =\left(\frac{\partial f}{\partial t}, w\right)-\zeta^{\prime \prime}\left(u_{0}, w\right)-\zeta \int_{\Omega} 2 \mu^{\prime}(\theta) \frac{\partial \theta}{\partial t} D\left(u_{0}\right): D(w) d x \\
& -\zeta^{\prime} \int_{\Omega} 2 \mu(\theta) D\left(u_{0}\right): D(w) d x-\zeta^{\prime} b\left(u_{0}, v_{\varepsilon m}^{\delta}, w\right)-\zeta b\left(u_{0}, \frac{\partial v_{\varepsilon m}^{\delta}}{\partial t}, w\right) \\
& -2 \zeta \zeta^{\prime} b\left(u_{0}, u_{0}, w\right)-\zeta^{\prime} b\left(v_{\varepsilon m}^{\delta}, u_{0}, w\right)-\zeta b\left(\frac{\partial v_{\varepsilon m}^{\delta}}{\partial t}, u_{0}, w\right) \text { a.e. in }(0, T),
\end{aligned}
$$

for all $w \in \operatorname{Span}\left\{w_{1}, \ldots, w_{m}\right\}$. Next we choose $w=\frac{\partial v_{\varepsilon m}^{\delta}}{\partial t}$ and we observe that

$$
\int_{\Gamma_{0}} \ell\left(\frac{\left(\frac{\partial v_{\varepsilon m}^{\delta}}{\partial t}\right)^{2}}{\sqrt{\varepsilon^{2}+\left|v_{\varepsilon m}^{\delta}\right|^{2}}}-\frac{\left(v_{\varepsilon m}^{\delta} \cdot \frac{\partial v_{\varepsilon m}^{\delta}}{\partial t}\right)^{2}}{\left(\varepsilon^{2}+\left|v_{\varepsilon m}^{\delta}\right|^{2}\right)^{3 / 2}}\right) d x^{\prime} \geq \int_{\Gamma_{0}} \ell \varepsilon^{2} \frac{\left(\frac{\partial v_{\varepsilon m}^{\delta}}{\partial t}\right)^{2}}{\left(\varepsilon^{2}+\left|v_{\varepsilon m}^{\delta}\right|^{2}\right)^{3 / 2}} d x^{\prime} \geq 0
$$

and

$$
b\left(v_{\varepsilon m}^{\delta}, \frac{\partial v_{\varepsilon m}^{\delta}}{\partial t}, \frac{\partial v_{\varepsilon m}^{\delta}}{\partial t}\right)+\frac{1}{2} \int_{\Omega} \operatorname{div}\left(\widetilde{v}_{\varepsilon m}^{\delta}\right) \frac{\partial \widetilde{v}_{\varepsilon m}^{\delta}}{\partial t} \cdot \frac{\partial v_{\varepsilon m}^{\delta}}{\partial t} d x=0, \quad b\left(u_{0}, \frac{\partial v_{\varepsilon m}^{\delta}}{\partial t}, \frac{\partial v_{\varepsilon m}^{\delta}}{\partial t}\right)=0
$$

Moreover with Lemma 4.1 in [4] we have

$$
\begin{aligned}
& \left\|v_{\varepsilon m}^{\delta}(s)\right\|_{\mathbf{L}^{2}(\Omega)}^{2}+\frac{2}{\delta} \int_{0}^{s}\left\|\operatorname{div}\left(v_{\varepsilon m}^{\delta}\right)\right\|_{L^{2}(\Omega)}^{2} d t+\alpha \int_{0}^{s}\left\|v_{\varepsilon m}^{\delta}\right\|_{\mathbf{H}^{1}(\Omega)}^{2} d s \\
& \leq C_{1}^{\prime} \exp \left(C_{1}^{\prime \prime} s\right) \quad \forall s \in[0, T]
\end{aligned}
$$

Then, by using Young's inequalities and Cauchy-Schwarz's inequality we obtain

$$
\begin{aligned}
& \left\|\frac{\partial v_{\varepsilon m}^{\delta}}{\partial t}(s)\right\|_{\mathbf{L}^{2}(\Omega)}^{2}+\frac{2}{\delta} \int_{0}^{s}\left\|\operatorname{div}\left(\frac{\partial v_{\varepsilon m}^{\delta}}{\partial t}\right)\right\|_{L^{2}(\Omega)}^{2} d t \\
& +\left(\alpha-3 K^{2} \sqrt{\frac{\mathcal{D}}{\alpha}}\right) \int_{0}^{s}\left\|\frac{\partial v_{\varepsilon m}^{\delta}}{\partial t}\right\|_{\mathbf{H}^{1}(\Omega)}^{2} d t \leq \mathbf{A}_{\mathbf{4}} \exp \left(2 \mathbf{A}_{\mathbf{3}} s\right) \quad \forall s \in[0, T]
\end{aligned}
$$

(for the details the reader is referred to Proposition 4.1 and Proposition 4.2 in [2]). 
Now let $\varphi \in \mathbf{H}_{0 \text { div }}^{1}(\Omega)$. For all $m \geq 1$ we define $\varphi_{m}$ as the orthogonal projection of $\varphi$ with respect to the inner product of $\mathbf{H}^{1}(\Omega)$ on $\operatorname{Span}\left\{w_{1}, \cdots, w_{m}\right\}$. Then for all $m \geq 1$ and $\chi \in \mathcal{D}(0, T)$, and recalling (11), we get

$$
\begin{aligned}
& -\int_{0}^{T}\left(\frac{\partial v_{\varepsilon m}^{\delta}}{\partial t}, \varphi_{m}\right) \chi^{\prime} d t-\int_{0}^{T}\left(b\left(\frac{\partial v_{\varepsilon m}^{\delta}}{\partial t}, \varphi_{m}, v_{\varepsilon m}^{\delta}\right)+b\left(v_{\varepsilon m}^{\delta}, \varphi_{m}, \frac{\partial v_{\varepsilon m}^{\delta}}{\partial t}\right)\right) \chi d t \\
& +\frac{1}{2} \int_{0}^{T} \int_{\Omega} \operatorname{div}\left(v_{\varepsilon m}^{\delta}\right) v_{\varepsilon m}^{\delta} \cdot \varphi_{m} \chi^{\prime} d x d t \\
& +\frac{1}{\delta} \int_{0}^{T}\left(\operatorname{div}\left(\frac{\widetilde{v}_{\varepsilon m}^{\delta}}{\partial t}\right), \operatorname{div}\left(\varphi_{m}\right)\right) \chi d t \\
& +\int_{0}^{T}\left(\int_{\Omega} 2 \mu^{\prime}(\theta) \frac{\partial \theta}{\partial t} D\left(v_{\varepsilon m}^{\delta}\right): D\left(\varphi_{m}\right) d x+a\left(\theta ; \frac{\partial v_{\varepsilon m}^{\delta}}{\partial t}, \varphi_{m}\right)\right) \chi d t \\
& +\int_{0}^{T} \int_{\Gamma_{0}} \frac{\partial \ell}{\partial t} \frac{v_{\varepsilon m}^{\delta} \cdot \varphi_{m}}{\sqrt{\varepsilon^{2}+\left|v_{\varepsilon m}^{\delta}\right|^{2}}} \chi d x^{\prime} d t \\
& +\int_{0}^{T} \int_{\Gamma_{0}} \ell\left(\frac{\frac{\partial v_{\varepsilon m}^{\delta}}{\partial t} \cdot \varphi_{m}}{\sqrt{\varepsilon^{2}+\left|v_{\varepsilon m}^{\delta}\right|^{2}}}-\frac{\left(v_{\varepsilon m}^{\delta} \cdot \frac{\partial v_{\varepsilon m}^{\delta}}{\partial t}\right)\left(v_{\varepsilon m}^{\delta} \cdot \varphi_{m}\right)}{\left(\varepsilon^{2}+\left|v_{\varepsilon m}^{\delta}\right|^{2}\right)^{3 / 2}}\right) \chi d x^{\prime} d t \\
& =\int_{0}^{T}\left(\left(\frac{\partial f}{\partial t}, \varphi_{m}\right)-\zeta^{\prime \prime}\left(u_{0}, \varphi_{m}\right)\right) \chi d t-\int_{0}^{T} \int_{\Omega} 2 \zeta \mu^{\prime}(\theta) \frac{\partial \theta}{\partial t} D\left(u_{0}\right): D\left(\varphi_{m}\right) \chi d x d t \\
& -\int_{0}^{T} \int_{\Omega} 2 \zeta^{\prime} \mu(\theta) D\left(u_{0}\right): D\left(\varphi_{m}\right) \chi d x d t-\int_{0}^{T} \zeta^{\prime} b\left(u_{0}, v_{\varepsilon m}^{\delta}, \varphi_{m}\right) \chi d t \\
& -\int_{0}^{T} \zeta b\left(u_{0}, \frac{\partial v_{\varepsilon m}^{\delta}}{\partial t}, \varphi_{m}\right) \chi d t-\int_{0}^{T} 2 \zeta \zeta^{\prime} b\left(u_{0}, u_{0}, \varphi_{m}\right) \chi d t \\
& -\int_{0}^{T} \zeta^{\prime} b\left(v_{\varepsilon m}^{\delta}, u_{0}, \varphi_{m}\right) \chi d t-\int_{0}^{T} \zeta b\left(\frac{\partial v_{\varepsilon m}^{\delta}}{\partial t}, u_{0}, \varphi_{m}\right) \chi d t
\end{aligned}
$$

Using the continuity of the trace operator from $\mathbf{H}^{1}(\Omega)$ into $\mathbf{L}^{4}(\partial \Omega)$ (see Theorem 1.23 page 17 in [26]) we obtain that

$$
\left|\int_{0}^{T} \frac{\partial \ell}{\partial t} \frac{v_{\varepsilon m}^{\delta} \cdot \varphi_{m}}{\sqrt{\varepsilon^{2}+\left|v_{\varepsilon m}^{\delta}\right|^{2}}} \chi d x^{\prime} d t\right| \leq\left\|\frac{\partial \ell}{\partial t}\right\|_{L^{2}\left(0, T ; \mathbf{L}^{2}\left(\Gamma_{0}\right)\right)}\left\|\varphi_{m}\right\|_{\mathbf{L}^{2}\left(\Gamma_{0}\right)}\|\chi\|_{L^{2}(0, T ; \mathbb{R})} \rightarrow_{m \rightarrow+\infty} 0
$$

and

$$
\begin{aligned}
& \left|\int_{0}^{T} \int_{\Gamma_{0}} \ell\left(\frac{\frac{\partial v_{\varepsilon m}^{\delta}}{\partial t} \cdot \varphi_{m}}{\sqrt{\varepsilon^{2}+\left|v_{\varepsilon m}^{\delta}\right|^{2}}}-\frac{\left(v_{\varepsilon m}^{\delta} \cdot \frac{\partial v_{\varepsilon m}^{\delta}}{\partial t}\right)\left(v_{\varepsilon m}^{\delta} \cdot \varphi_{m}\right)}{\left(\varepsilon^{2}+\left|v_{\varepsilon m}^{\delta}\right|^{2}\right)^{3 / 2}}\right) \chi d x^{\prime} d t\right| \\
& \leq \frac{2}{\varepsilon}\|\ell\|_{L^{2}\left(0, T ; \mathbf{L}^{2}\left(\Gamma_{0}\right)\right)}\left\|\frac{\partial v_{\varepsilon m}^{\delta}}{\partial t}\right\|_{L^{2}\left(0, T ; \mathbf{L}^{4}\left(\Gamma_{0}\right)\right)}\left\|\varphi_{m}\right\|_{\mathbf{L}^{4}\left(\Gamma_{0}\right)}\|\chi\|_{L^{\infty}(0, T ; \mathbb{R})} \rightarrow_{m \rightarrow+\infty} 0 .
\end{aligned}
$$

Then, by using the same compactness arguments as in [4] we may pass to the limit as $m$ tends to 
$+\infty$, then as $\delta$ tends to zero. We obtain

$$
\begin{aligned}
& -\int_{0}^{T}\left(\frac{\partial v_{\varepsilon}}{\partial t}, \varphi\right) \chi^{\prime} d t+\int_{0}^{T}\left(b\left(\frac{\partial v_{\varepsilon}}{\partial t}, v_{\varepsilon}, \varphi\right)+b\left(v_{\varepsilon}, \frac{\partial v_{\varepsilon}}{\partial t}, \varphi\right)\right) \chi d t \\
+ & \int_{0}^{T}\left(\int_{\Omega} 2 \mu^{\prime}(\theta) \frac{\partial \theta}{\partial t} D\left(v_{\varepsilon}\right): D(\varphi) d x+a\left(\theta ; \frac{\partial v_{\varepsilon}}{\partial t}, \varphi\right)\right) \chi d t \\
= & \int_{0}^{T}\left(\left(\frac{\partial f}{\partial t}, \varphi\right)-\zeta^{\prime \prime}\left(u_{0}, \varphi\right)\right) \chi d t-\int_{0}^{T} \int_{\Omega} 2 \zeta \mu^{\prime}(\theta) \frac{\partial \theta}{\partial t} D\left(u_{0}\right): D(\varphi) \chi d x d t \\
- & \int_{0}^{T} \int_{\Omega}^{T} 2 \zeta^{\prime} \mu(\theta) D\left(u_{0}\right): D(\varphi) \chi d x d t-\int_{0}^{T} \zeta^{\prime} b\left(u_{0}, v_{\varepsilon}, \varphi\right) \chi d t \\
- & \int_{0}^{T} \zeta b\left(u_{0}, \frac{\partial v_{\varepsilon}}{\partial t}, \varphi\right) \chi d t-\int_{0}^{T} 2 \zeta \zeta^{\prime} b\left(u_{0}, u_{0}, \varphi\right) \chi d t \\
- & \int_{0}^{T} \zeta^{\prime} b\left(v_{\varepsilon}, u_{0}, \varphi\right) \chi d t-\int_{0}^{T} \zeta b\left(\frac{\partial v_{\varepsilon}}{\partial t}, u_{0}, \varphi\right) \chi d t \quad \forall(\varphi, \chi) \in \mathbf{H}_{0 d i v}^{1}(\Omega) \times \mathcal{D}(0, T) .
\end{aligned}
$$

It follows that

$$
\begin{aligned}
& \left\langle\frac{\partial}{\partial t}\left(\frac{\partial v_{\varepsilon}}{\partial t}, \varphi\right), \chi\right\rangle+\int_{0}^{T}\left(b\left(\frac{\partial v_{\varepsilon}}{\partial t}, v_{\varepsilon}, \varphi\right)+b\left(v_{\varepsilon}, \frac{\partial v_{\varepsilon}}{\partial t}, \varphi\right)\right) \chi d t \\
& +\int_{0}^{T}\left(\int_{\Omega} 2 \mu^{\prime}(\theta) \frac{\partial \theta}{\partial t} D\left(v_{\varepsilon}\right): D(\varphi) d x+a\left(\theta ; \frac{\partial v_{\varepsilon}}{\partial t}, \varphi\right)\right) \chi d t \\
& =\int_{0}^{T}\left(\left(\frac{\partial f}{\partial t}, \varphi\right)-\zeta^{\prime \prime}\left(u_{0}, \varphi\right)\right) \chi d t-\int_{0}^{T} \int_{\Omega} 2 \zeta \mu^{\prime}(\theta) \frac{\partial \theta}{\partial t} D\left(u_{0}\right): D(\varphi) \chi d x d t \\
& -\int_{0}^{T} \int_{\Omega}^{T} 2 \zeta^{\prime} \mu(\theta) D\left(u_{0}\right): D(\varphi) \chi d x d t-\int_{0}^{T} \zeta^{\prime} b\left(u_{0}, v_{\varepsilon}, \varphi\right) \chi d t \\
& -\int_{0}^{T} \zeta b\left(u_{0}, \frac{\partial v_{\varepsilon}}{\partial t}, \varphi\right) \chi d t-\int_{0}^{T} 2 \zeta \zeta^{\prime} b\left(u_{0}, u_{0}, \varphi\right) \chi d t \\
& -\int_{0}^{T} \zeta^{\prime} b\left(v_{\varepsilon}, u_{0}, \varphi\right) \chi d t-\int_{0}^{T} \zeta b\left(\frac{\partial v_{\varepsilon}}{\partial t}, u_{0}, \varphi\right) \chi d t \quad \forall(\varphi, \chi) \in \mathbf{H}_{0 d i v}^{1}(\Omega) \times \mathcal{D}(0, T) .
\end{aligned}
$$

Using the previous estimates we obtain that $\frac{\partial^{2} v_{\varepsilon}}{\partial t^{2}} \in L^{2}\left(0, T,\left(\mathbf{H}_{0 d i v}^{1}(\Omega)\right)^{\prime}\right)$ and remains uniformly bounded with respect to $\varepsilon$ in $L^{2}\left(0, T,\left(\mathbf{H}_{0 \text { div }}^{1}(\Omega)\right)^{\prime}\right)$ which allows us to conclude.

Let us define

$$
F(\Omega)=\left\{\widetilde{\sigma} \in\left(L^{2}(\Omega)\right)^{3 \times 3}: \quad \operatorname{div}(\widetilde{\sigma}) \in \mathbf{L}^{4 / 3}(\Omega)\right\}
$$

endowed with the norm

$$
\|\widetilde{\sigma}\|_{F(\Omega)}=\left(\|\widetilde{\sigma}\|_{\left(L^{2}(\Omega)\right)^{3 \times 3}}^{2}+\|\operatorname{div}(\widetilde{\sigma})\|_{\mathbf{L}^{4 / 3}(\Omega)}^{2}\right)^{1 / 2} \quad \forall \widetilde{\sigma} \in F(\Omega) .
$$

By using the existence, uniqueness and regularity results for Tresca's problem, we have:

Proposition 3.4. Let us assume that $\ell \in H^{1}\left(0, T ; L_{+}^{2}\left(\Gamma_{0}\right)\right)$, (10), (14)-(17) hold and condition (26) is satisfied. Let $(v, p)$ be the unique regular solution of Tresca's problem and let $\sigma=-p I+$ 
$2 \mu(\theta) D\left(v+u_{0} \zeta\right)$. Then $\sigma \in L^{\infty}(0, T ; F(\Omega))$ and there exists a positive constant $C_{\text {data }}$, depending only on the data $\mu, \zeta, f, u_{0}$ and $\theta$, such that

$$
\|\sigma\|_{L^{\infty}(0, T ; F(\Omega))} \leq C_{\text {data }}\left(1+\left\|\frac{\partial \ell}{\partial t}\right\|_{L^{2}\left(0, T ; L^{2}\left(\Gamma_{0}\right)\right)}\right) .
$$

Proof. Starting from the previous results, we get immediately $\sigma \in L^{\infty}\left(0, T ;\left(L^{2}(\Omega)\right)^{3 \times 3}\right)$ and

$$
\begin{aligned}
& \left.\|\sigma\|_{L^{\infty}\left(0, T ;\left(L^{2}(\Omega)\right)^{3 \times 3}\right)} \leq\|p\|_{L^{\infty}\left(0, T ; L^{2}(\Omega)\right)}+\mu_{*}\left\|D\left(v+u_{0} \zeta\right)\right\|_{L^{\infty}\left(0, T ;\left(L^{2}(\Omega)\right)^{3 \times 3}\right)}\left(\left\|\frac{\partial v}{\partial t}\right\|_{L^{\infty}\left(0, T ; \mathbf{L}^{2}(\Omega)\right)}+K^{2}\left(\frac{\alpha}{3 K^{2}}+\left\|u_{0}\right\|_{\mathbf{H}^{1}(\Omega)}\|\zeta\|_{C([0, T])}\right)\right)^{2}\right) \\
& \leq\|P\|_{\mathcal{L}\left(L_{0}^{2}(\Omega), \mathbf{H}_{0}^{1}(\Omega)\right)}\left(\|\|_{*}\left(\|P\|_{\mathcal{L}\left(L_{0}^{2}(\Omega), \mathbf{H}_{0}^{1}(\Omega)\right)}+1\right)\left(\frac{\alpha}{3 K^{2}}+\left\|u_{0}\right\|_{\mathbf{H}^{1}(\Omega)}\|\zeta\|_{C([0, T])}\right)\right. \\
& +\|P\|_{\mathcal{L}\left(L_{0}^{2}(\Omega), \mathbf{H}_{0}^{1}(\Omega)\right)}\left(\|f\|_{L^{\infty}\left(0, T ; \mathbf{L}^{2}(\Omega)\right)}+\left\|u_{0}\right\|_{\mathbf{L}^{2}(\Omega)}\left\|\zeta^{\prime}\right\|_{C([0, T])}\right) .
\end{aligned}
$$

In order to estimate $\operatorname{div}(\sigma)$ we choose $\varphi \in(\mathcal{D}(\Omega))^{3}$ in $(22)$. We get

$$
\begin{aligned}
& \left\langle\frac{\partial}{\partial t}(v, \varphi), \pm \chi\right\rangle+\left\langle b\left(v+u_{0} \zeta, v+u_{0} \zeta, \varphi\right), \pm \chi\right\rangle-\langle(p, \operatorname{div}(\varphi)), \chi\rangle \\
& +\left\langle a\left(\theta ; v+u_{0} \zeta, \varphi\right), \pm \chi\right\rangle \geq\langle(f, \varphi), \pm \chi\rangle-\left\langle\zeta^{\prime}\left(u_{0}, \varphi\right), \pm \chi\right\rangle
\end{aligned}
$$

for all $\chi \in \mathcal{D}(0, T)$. It follows that

$$
\begin{aligned}
& \left\langle\frac{\partial}{\partial t}(v, \varphi), \chi\right\rangle+\left\langle b\left(v+u_{0} \zeta, v+u_{0} \zeta, \varphi\right), \chi\right\rangle+\int_{0}^{T} \int_{\Omega} \sum_{i, j=1}^{3} \sigma_{i j} \frac{\partial \varphi_{i}}{\partial x_{j}} \chi d x d t \\
& =\langle(f, \varphi), \chi\rangle-\left\langle\zeta^{\prime}\left(u_{0}, \varphi\right), \chi\right\rangle
\end{aligned}
$$

and thus

$$
\begin{aligned}
& \left|\int_{0}^{T} \int_{\Omega} \sigma_{i j} \frac{\partial \varphi_{i}}{\partial x_{j}} \chi d x d t\right| \\
& \leq\left(\left\|\frac{\partial v}{\partial t}\right\|_{L^{\infty}\left(0, T ; \mathbf{L}^{2}(\Omega)\right)}+\|f\|_{L^{\infty}\left(0, T ; \mathbf{L}^{2}(\Omega)\right)}+\left\|u_{0}\right\|_{\mathbf{L}^{2}(\Omega)}\left\|\zeta^{\prime}\right\|_{C([0, T])}\right)\|\varphi \chi\|_{L^{1}\left(0, T ; \mathbf{L}^{2}(\Omega)\right)}\|\varphi \chi\|_{L^{1}\left(0, T ; \mathbf{L}^{4}(\Omega)\right)} \\
& \left.+K\left\|v+u_{0} \zeta\right\|_{L^{\infty}\left(0, T ; \mathbf{H}^{1}(\Omega)\right)}+\|f\|_{L^{\infty}\left(0, T ; \mathbf{L}^{2}(\Omega)\right)}+\left\|u_{0}\right\|_{\mathbf{L}^{2}(\Omega)}\left\|\zeta^{\prime}\right\|_{C([0, T])}\right) \\
& \leq\left(|\Omega|^{\frac{1}{4}}\left(\left\|\frac{\partial v}{\partial t}\right\|_{L^{\infty}\left(0, T ; \mathbf{L}^{2}(\Omega)\right)}+\|\|^{2}\right)^{2}\|\varphi \chi\|_{L^{1}\left(0, T ; \mathbf{L}^{4}(\Omega)\right)} .\right.
\end{aligned}
$$

Hence $\operatorname{div}(\sigma) \in\left(L^{1}\left(0, T ; \mathbf{L}^{4}(\Omega)\right)\right)^{\prime}=L^{\infty}\left(0, T ; \mathbf{L}^{\frac{4}{3}}(\Omega)\right)$ and

$$
\begin{aligned}
& \|\operatorname{div}(\sigma)\|_{L^{\infty}\left(0, T ; \mathbf{L}^{\frac{4}{3}}(\Omega)\right.} \leq|\Omega|^{\frac{1}{4}}\left(\left\|\frac{\partial v}{\partial t}\right\|_{L^{\infty}\left(0, T ; \mathbf{L}^{2}(\Omega)\right)}+\|f\|_{L^{\infty}\left(0, T ; \mathbf{L}^{2}(\Omega)\right)}+\left\|u_{0}\right\|_{\mathbf{L}^{2}(\Omega)}\left\|\zeta^{\prime}\right\|_{C([0, T])}\right) \\
& +K\left(\frac{\alpha}{3 K^{2}}+\left\|u_{0}\right\|_{\mathbf{H}^{1}(\Omega)}\|\zeta\|_{C([0, T])}\right)^{2} .
\end{aligned}
$$

Then with (28) we may conclude with

$$
C_{\text {data }}=\underset{14}{2 \max \left(\widetilde{C}_{\text {data }}, \bar{C}_{\text {data }}\right)}
$$


where

$$
\begin{aligned}
& \widetilde{C}_{\text {data }}=\left(\|P\|_{\mathcal{L}\left(L_{0}^{2}(\Omega), \mathbf{H}_{0}^{1}(\Omega)\right)}+|\Omega|^{\frac{1}{4}}\right) \frac{\sqrt{7} \gamma(\Omega)}{\sqrt{\alpha}} \exp \left(\mathbf{A}_{\mathbf{3}} T\right) \\
& \bar{C}_{\text {data }}=\left(\|P\|_{\mathcal{L}\left(L_{0}^{2}(\Omega), \mathbf{H}_{0}^{1}(\Omega)\right)}+|\Omega|^{\frac{1}{4}}\right)\left(\sqrt{\mathbf{A}_{4}^{\prime}} \exp \left(\mathbf{A}_{\mathbf{3}} T\right)\right. \\
& \left.+\left(K+K^{2}\right)\left(\frac{\alpha}{3 K^{2}}+\left\|u_{0}\right\|_{\mathbf{H}^{1}(\Omega)}\|\zeta\|_{\mathcal{C}([0, T])}\right)^{2}+\|f\|_{L^{\infty}\left(0, T ; \mathbf{L}^{2}(\Omega)\right)}+\left\|u_{0}\right\|_{\mathbf{L}^{2}(\Omega)}\left\|\zeta^{\prime}\right\|_{\mathcal{C}([0, T])}\right) \\
& +\mu_{*}\left(\|P\|_{\mathcal{L}\left(L_{0}^{2}(\Omega), \mathbf{H}_{0}^{1}(\Omega)\right)}+1\right)\left(\frac{\alpha}{3 K^{2}}+\left\|u_{0}\right\|_{\mathbf{H}^{1}(\Omega)}\|\zeta\|_{C([0, T])}\right)
\end{aligned}
$$

Remark 3.2. With (39) we get

$$
\begin{aligned}
& \int_{0}^{T}\langle\operatorname{div}(\sigma), \varphi\rangle_{\left(\mathcal{D}^{\prime}(\Omega)\right)^{3},(\mathcal{D}(\Omega))^{3}} \chi d t=\int_{0}^{T}\langle\operatorname{div}(\sigma), \varphi\rangle_{\mathbf{L}^{4 / 3}(\Omega), \mathbf{L}^{4}(\Omega)} \chi d t \\
& =\left\langle\left(\frac{\partial v}{\partial t}, \varphi\right), \chi\right\rangle+\left\langle b\left(v+u_{0} \zeta, v+u_{0} \zeta, \varphi\right), \chi\right\rangle \\
& -\langle(f, \varphi), \chi\rangle+\left\langle\zeta^{\prime}\left(u_{0}, \varphi\right), \chi\right\rangle \quad \forall \varphi \in(\mathcal{D}(\Omega))^{3}, \forall \chi \in \mathcal{D}(0, T)
\end{aligned}
$$

and by density of $(\mathcal{D}(\Omega))^{3}$ into $\mathbf{L}^{4}(\Omega)$, the same equality holds for all $\varphi \in \mathbf{L}^{4}(\Omega)$ and for all $\chi \in \mathcal{D}(0, T)$. Hence, for all $\varphi \in \mathbf{L}^{4}(\Omega)$, we have

$$
\begin{aligned}
& \langle\operatorname{div}(\sigma), \varphi\rangle_{\mathbf{L}^{4 / 3}(\Omega), \mathbf{L}^{4}(\Omega)} \\
& =\left(\frac{\partial v}{\partial t}, \varphi\right)+b\left(v+u_{0} \zeta, v+u_{0} \zeta, \varphi\right)-(f, \varphi)+\zeta^{\prime}\left(u_{0}, \varphi\right) \quad \text { in } L^{\infty}(0, T) .
\end{aligned}
$$

\section{Existence for Coulomb's problem (P)}

In order to prove the existence of a solution to problem $(P)$ we will apply now a successive approximation technique with respect to the friction threshold.

First we observe that, for any $\sigma \in L^{\infty}(0, T ; F(\Omega))$ we may define $\mathcal{F}\left(\cdot, \cdot, \sigma_{n}\right)$. Indeed,

Proposition 4.1. The set $(\mathcal{D}(\bar{\Omega}))^{3 \times 3}$ is dense in $F(\Omega)$ and there exists a linear continuous operator $\gamma_{n} \in \mathcal{L}\left(F(\Omega), \mathbf{H}^{-1 / 2}(\partial \Omega)\right)$ such that

$$
\gamma_{n}(\widetilde{\sigma})=\widetilde{\sigma} \cdot n \quad \text { on } \partial \Omega \text { for any } \widetilde{\sigma} \in(\mathcal{D}(\bar{\Omega}))^{3 \times 3} .
$$

Moreover the following Stokes formula remains true for all $\widetilde{\sigma} \in F(\Omega)$ and $w \in \mathbf{H}^{1}(\Omega)$

$$
(\widetilde{\sigma}, \operatorname{grad} w)_{\left(L^{2}(\Omega)\right)^{3 \times 3}}+\langle\operatorname{div}(\widetilde{\sigma}), w\rangle_{\mathbf{L}^{4 / 3}(\Omega), \mathbf{L}^{4}(\Omega)}=\left\langle\gamma_{n}(\widetilde{\sigma}), w\right\rangle_{\mathbf{H}^{-1 / 2}(\partial \Omega), \mathbf{H}^{1 / 2}(\partial \Omega)}
$$

where $(\cdot, \cdot)_{\left(L^{2}(\Omega)\right)^{3 \times 3}}$ denotes the inner product in $\left(L^{2}(\Omega)\right)^{3 \times 3}$.

Proof. We apply the same arguments as in the proof of Theorem 1.1 in [30] and Theorem 1.2.5 in [16]. 
For the sake of notational simplicity we will denote simply $\widetilde{\sigma}_{n}$ instead of $\gamma_{n}(\widetilde{\sigma})$ for any $\widetilde{\sigma} \in F(\Omega)$. Then, following $[10,11,8]$ we introduce a regularization operator $\mathcal{R}$ by using a convolution technique which fits the mechanical meaning of the normal component of the stress tensor that is defined as the ratio of a force by a surface. More precisely, for any $\widetilde{\sigma} \in F(\Omega)$ we define $\mathcal{R}\left(\widetilde{\sigma}_{n}\right) \in \mathcal{C}\left(\Gamma_{0}\right)$ by

$$
\mathcal{R}\left(\widetilde{\sigma}_{n}\right)\left(x^{\prime}\right)=\left\langle\widetilde{\sigma}_{n}, \tilde{\varphi}_{x^{\prime}}\right\rangle_{\mathbf{H}^{-1 / 2}(\partial \Omega), \mathbf{H}^{1 / 2}(\partial \Omega)} \quad \forall x^{\prime} \in \Gamma_{0}
$$

where $\tilde{\varphi}$ is a function belonging to $\mathcal{D}\left(\Gamma_{0} \times \mathbb{R}^{3} ; \mathbb{R}^{3}\right)$ and $\tilde{\varphi}_{x^{\prime}}: \Omega \rightarrow \mathbb{R}^{3}$ is defined by $\tilde{\varphi}_{x^{\prime}}(x)=$ $\tilde{\varphi}\left(x^{\prime}, x^{\prime}-x\right)$ for all $x \in \Omega$ and for all $x^{\prime} \in \Gamma_{0}$. It follows that $\mathcal{R} \in \mathcal{L}_{c}\left(F(\Omega) ; C\left(\Gamma_{0}\right)\right)$ and we have:

Proposition 4.2. Let $\sigma \in L^{\infty}(0, T ; F(\Omega))$. Let us recall (see (9) and (13)) that

$$
\mathcal{F}\left(t, x^{\prime}, \sigma_{n}\right)=k(t) \int_{0}^{t} S(t-s)\left|\mathcal{R}\left(\sigma_{n}(s, \cdot)\right)\left(x^{\prime}\right)\right| d s \quad \forall x^{\prime} \in \Gamma_{0}, \forall t \in[0, T]
$$

where $S \in C^{1}\left(\mathbb{R}^{+} ; \mathbb{R}^{+}\right)$and $k \in W^{1, \infty}\left(0, T ; \mathbb{R}^{+}\right)$. Then $\mathcal{F}\left(\cdot, \cdot, \sigma_{n}\right) \in W^{1, \infty}\left(0, T ; L^{2}\left(\Gamma_{0}\right)\right)$ and there exists a positive constant $C_{\text {data }}^{\prime}$, depending only on $\|\mathcal{R}\|_{\mathcal{L}_{c}\left(F(\Omega) ; C\left(\Gamma_{0}\right)\right)}$ and on the data $S$ and $k$, such that

$$
\left\|\mathcal{F}\left(\cdot, \cdot, \sigma_{n}\right)\right\|_{W^{1, \infty}\left(0, T ; L^{2}\left(\Gamma_{0}\right)\right)} \leq C_{\text {data }}^{\prime}\|\sigma\|_{L^{\infty}(0, T ; F(\Omega))} .
$$

Proof. Since $\mathcal{R}\left(\sigma_{n}\right) \in L^{\infty}\left(0, T ; C\left(\Gamma_{0}\right)\right)$, it is straighforward that $\mathcal{F}\left(\cdot, \cdot, \sigma_{n}\right) \in W^{1, \infty}\left(0, T ; L^{2}\left(\Gamma_{0}\right)\right)$ and

$$
\begin{aligned}
& \left\|\mathcal{F}\left(\cdot, \cdot, \sigma_{n}\right)\right\|_{L^{\infty}\left(0, T ; L^{2}\left(\Gamma_{0}\right)\right)} \\
& \leq T\|k\|_{L^{\infty}(0, T)}\|S\|_{C([0, T])}\|\mathcal{R}\|_{\mathcal{L}_{c}\left(F(\Omega) ; C\left(\Gamma_{0}\right)\right)}\left|\Gamma_{0}\right|^{1 / 2}\|\sigma\|_{L^{\infty}(0, T ; F(\Omega))}
\end{aligned}
$$

Moreover

$$
\begin{aligned}
& \frac{\partial\left(\mathcal{F}\left(\cdot, \cdot, \sigma_{n}\right)\right)}{\partial t}(t, \cdot)=k^{\prime}(t) \int_{0}^{t} S(t-s)\left|\mathcal{R}\left(\sigma_{n}(s, \cdot)\right)(\cdot)\right| d s+k(t) S(0)\left|\mathcal{R}\left(\sigma_{n}(t, \cdot)\right)(\cdot)\right| \\
& +k(t) \int_{0}^{t} S^{\prime}(t-s)\left|\mathcal{R}\left(\sigma_{n}(s, \cdot)\right)(\cdot)\right| d s \quad \forall \text { a.e. } t \in[0, T] .
\end{aligned}
$$

Then

$$
\begin{aligned}
& \left\|\frac{\partial\left(\mathcal{F}\left(\cdot, \cdot, \sigma_{n}\right)\right)}{\partial t}(t, \cdot)\right\|_{L^{2}\left(\Gamma_{0}\right)} \leq\left|k^{\prime}(t)\right| \int_{0}^{t}|S(t-s)|\left\|\mathcal{R}\left(\sigma_{n}(s, \cdot)\right)\right\|_{L^{2}\left(\Gamma_{0}\right)} d s \\
& +\left|k(t)\left\|S(0)\left|\left\|\mathcal{R}\left(\sigma_{n}(t, \cdot)\right)\right\|_{L^{2}\left(\Gamma_{0}\right)}+\right| k(t)\left|\int_{0}^{t}\right| S^{\prime}(t-s) \mid\right\| \mathcal{R}\left(\sigma_{n}(s, \cdot)\right) \|_{L^{2}\left(\Gamma_{0}\right)} d s\right. \\
& \leq\left(T\left\|k^{\prime}\right\|_{L^{\infty}(0, T)}\|S\|_{C([0, T])}+T\|k\|_{L^{\infty}(0, T)}\left\|S^{\prime}\right\|_{C([0, T])}+\|k\|_{L^{\infty}(0, T)}\|S\|_{C([0, T])}\right) \\
& \times\|\mathcal{R}\|_{\mathcal{L}_{c}\left(F(\Omega) ; C\left(\Gamma_{0}\right)\right)}\left|\Gamma_{0}\right|^{1 / 2}\|\sigma\|_{L^{\infty}(0, T ; F(\Omega))} \quad \forall \text { a.e. } t \in[0, T]
\end{aligned}
$$

and we may conclude with

$$
\begin{aligned}
C_{\text {data }}^{\prime}= & \max (T, 1)\left(\left\|k^{\prime}\right\|_{L^{\infty}(0, T)}\|S\|_{C([0, T])}+\|k\|_{L^{\infty}(0, T)}\left\|S^{\prime}\right\|_{C([0, T])}+\|k\|_{L^{\infty}(0, T)}\|S\|_{C([0, T])}\right) \\
& \times\|\mathcal{R}\|_{\mathcal{L}_{c}\left(F(\Omega) ; C\left(\Gamma_{0}\right)\right)}\left|\Gamma_{0}\right|^{1 / 2}
\end{aligned}
$$


Now let $\tau_{*} \in[0, T), \tau \in\left(\tau_{*}, T\right]$ and $\ell_{*} \in H^{1}\left(0, \tau_{*} ; L_{+}^{2}\left(\Gamma_{0}\right)\right)$. Possibly modifying $\ell_{*}$ on a negligible subset of $\left[0, \tau_{*}\right]$ we may assume without loss of generality that $\ell_{*} \in C\left(\left[0, \tau_{*}\right] ; L_{+}^{2}\left(\Gamma_{0}\right)\right)$. For any $L \in L^{2}\left(\tau_{*}, \tau ; L_{+}^{2}\left(\Gamma_{0}\right)\right)$ we define $\ell \in H^{1}\left(0, T ; L_{+}^{2}\left(\Gamma_{0}\right)\right)$ by

$$
\ell(t, \cdot)=\left\{\begin{array}{lll}
\ell_{*}(t, \cdot) \quad \text { on } \Gamma_{0} \times\left[0, \tau_{*}\right], & \\
\ell_{*}\left(\tau_{*}, \cdot\right)+\int_{\tau_{*}}^{t} L(s, \cdot) d s \quad \text { on } & \Gamma_{0} \times\left[\tau_{*}, \tau\right], \\
\ell_{*}\left(\tau_{*}, \cdot\right)+\int_{\tau_{*}}^{\tau} L(s, \cdot) d s & \text { on } & \Gamma_{0} \times[\tau, T] .
\end{array}\right.
$$

The corresponding Tresca's problem admits a unique regular solution on $[0, T]$, denoted as $\left(v^{\ell}, p^{\ell}\right)$, if condition (26) holds. Let us define

$$
\mathcal{D}_{0}^{\prime}=\mathcal{D}_{0}+\left(\mathbf{A}_{\mathbf{0}}^{\mathbf{2}}+2\left\|\frac{\partial f}{\partial t}\right\|_{L^{2}\left(0, T ; \mathbf{L}^{2}(\Omega)\right)}^{2}+2 \mathbf{A}_{\mathbf{1}} T+2 \mathbf{A}_{2} C_{1}^{2}\right) \exp \left(2 \mathbf{A}_{\mathbf{3}} T\right)
$$

where $\mathcal{D}_{0}$ and $\mathbf{A}_{\mathbf{i}}$ for $i \in\{0, \ldots, 3\}$ are defined as in Section 3. Then $\mathcal{D}_{0}^{\prime}$ is a positive constant depending only on the data $\mu, \zeta, f, \theta$ and $u_{0}$, and condition (26) is obviously satisfied if

$$
\mathcal{D}_{0}^{\prime}+\frac{7 \gamma(\Omega)^{2}}{\alpha}\left(\left\|\frac{\partial \ell_{*}}{\partial t}\right\|_{L^{2}\left(0, \tau_{*} ; L^{2}\left(\Gamma_{0}\right)\right)}^{2}+\left\|\frac{\partial \ell}{\partial t}\right\|_{L^{2}\left(\tau_{*}, \tau ; L^{2}\left(\Gamma_{0}\right)\right)}^{2}\right) \exp \left(2 \mathbf{A}_{\mathbf{3}} T\right)<\frac{\alpha^{3}}{9 K^{4}} .
$$

We will assume that

$$
\mathcal{D}_{0}^{\prime}+\frac{7 \gamma(\Omega)^{2}}{\alpha}\left\|\frac{\partial \ell_{*}}{\partial t}\right\|_{L^{2}\left(0, \tau_{*} ; L^{2}\left(\Gamma_{0}\right)\right)}^{2} \exp \left(2 \mathbf{A}_{3} T\right)<\frac{\alpha^{3}}{9 K^{4}}
$$

Then, for any $L \in L^{2}\left(\tau_{*}, \tau ; L_{+}^{2}\left(\Gamma_{0}\right)\right)$ such that

$$
\begin{aligned}
& \frac{7 \gamma(\Omega)^{2}}{\alpha}\|L\|_{L^{2}\left(\tau_{*}, \tau ; L^{2}\left(\Gamma_{0}\right)\right)}^{2} \exp \left(2 \mathbf{A}_{\mathbf{3}} T\right) \\
& \leq \frac{\alpha^{3}}{9 K^{4}}-\left(\mathcal{D}_{0}^{\prime}+\frac{7 \gamma(\Omega)^{2}}{\alpha}\left\|\frac{\partial \ell_{*}}{\partial t}\right\|_{L^{2}\left(0, \tau_{*} ; L^{2}\left(\Gamma_{0}\right)\right)}^{2} \exp \left(2 \mathbf{A}_{\mathbf{3}} T\right)\right)
\end{aligned}
$$

we may define $\tilde{\ell} \in W^{1, \infty}\left(0, T ; L_{+}^{2}\left(\Gamma_{0}\right)\right)$ by

$$
\tilde{\ell}\left(t, x^{\prime}\right)=\mathcal{F}\left(t, x^{\prime}, \sigma_{n}^{\ell}\right)=k(t) \int_{0}^{t} S(t-s)\left|\mathcal{R}\left(\sigma_{n}^{\ell}(s, \cdot)\right)\left(x^{\prime}\right)\right| d s \quad \forall x^{\prime} \in \Gamma_{0}, \forall t \in[0, T],
$$

with $\sigma^{\ell}=-p^{\ell} I+2 \mu(\theta) D\left(v^{\ell}+u_{0} \zeta\right)$ and we let $\Sigma(L)$ be the restriction of $\frac{\partial \tilde{\ell}}{\partial t}$ to the time interval $\left[\tau_{*}, \tau\right]$

By using Proposition 3.4 and Proposition 4.2 we get

$$
\begin{aligned}
& \|\tilde{\ell}\|_{W^{1, \infty}\left(0, T ; L^{2}\left(\Gamma_{0}\right)\right)} \leq C_{\text {data }}^{\prime} C_{\text {data }}\left(1+\left\|\frac{\partial \ell}{\partial t}\right\|_{L^{2}\left(0, T ; L^{2}\left(\Gamma_{0}\right)\right)}\right) \\
& \leq C_{\text {data }}^{\prime} C_{\text {data }}\left(1+\left(\left\|\frac{\partial \ell_{*}}{\partial t}\right\|_{L^{2}\left(0, \tau_{*}: L^{2}\left(\Gamma_{0}\right)\right)}^{2}+\|L\|_{L^{2}\left(\tau_{*}, \tau ; L^{2}\left(\Gamma_{0}\right)\right)}^{2}\right)^{1 / 2}\right) \\
& \leq C_{\text {data }}^{\prime} C_{\text {data }}\left(1+\frac{\sqrt{\alpha} \exp \left(-\mathbf{A}_{3} T\right)}{\sqrt{7} \gamma(\Omega)}\left(\frac{\alpha^{3}}{9 K^{4}}-\mathcal{D}_{0}^{\prime}\right)^{1 / 2}\right)
\end{aligned}
$$


Hence

$$
\|\Sigma(L)\|_{L^{2}\left(\tau_{*}, \tau ; L^{2}\left(\Gamma_{0}\right)\right)} \leq C_{\text {data }}^{\prime} C_{\text {data }} \sqrt{\tau-\tau_{*}}\left(1+\frac{\sqrt{\alpha} \exp \left(-\mathbf{A}_{\mathbf{3}} T\right)}{\sqrt{7} \gamma(\Omega)}\left(\frac{\alpha^{3}}{9 K^{4}}-\mathcal{D}_{0}^{\prime}\right)^{1 / 2}\right)
$$

and there exists $\rho>0$ such that, for any $\tau \in\left(\tau_{*}, \tau_{*}+\rho\right), \Sigma(L)$ satisfies (46) i.e.

$$
\begin{aligned}
& \frac{7 \gamma(\Omega)^{2}}{\alpha}\|\Sigma(L)\|_{L^{2}\left(\tau_{*}, \tau ; L^{2}\left(\Gamma_{0}\right)\right)}^{2} \exp \left(2 \mathbf{A}_{3} T\right) \\
& \leq \frac{\alpha^{3}}{9 K^{4}}-\left(\mathcal{D}_{0}^{\prime}+\frac{7 \gamma(\Omega)^{2}}{\alpha}\left\|\frac{\partial \ell_{*}}{\partial t}\right\|_{L^{2}\left(0, \tau_{*} ; L^{2}\left(\Gamma_{0}\right)\right)}^{2} \exp \left(2 \mathbf{A}_{\mathbf{3}} T\right)\right) .
\end{aligned}
$$

Thus, for any $\tau \in\left(\tau_{*}, \tau_{*}+\rho\right), \Sigma$ maps $\mathcal{B}=\left\{L \in L^{2}\left(\tau_{*}, \tau ; L_{+}^{2}\left(\Gamma_{0}\right)\right)\right.$ satisfying (46) $\}$ into itself.

So, starting from any $L_{0} \in \mathcal{B}$, we may construct by induction a bounded sequence $\left(L_{m}\right)_{m \geq 0}$ of $\mathcal{B}$ such that $L_{m+1}=\Sigma\left(L_{m}\right)$ for all $m \geq 0$. For all $m \geq 0$, we let

$$
\ell_{m}(t, \cdot)=\left\{\begin{array}{lll}
\ell_{*}(t, \cdot) \quad \text { on } \Gamma_{0} \times\left[0, \tau_{*}\right] & \\
\ell_{*}\left(\tau_{*}, \cdot\right)+\int_{\tau_{*}}^{t} L_{m}(s, \cdot) d s \quad \text { on } & \Gamma_{0} \times\left[\tau_{*}, \tau\right] \\
\ell_{*}\left(\tau_{*}, \cdot\right)+\int_{\tau_{*}}^{\tau} L_{m}(s, \cdot) d s & \text { on } \quad \Gamma_{0} \times[\tau, T]
\end{array}\right.
$$

we denote as $\left(v^{\ell_{m}}, p^{\ell_{m}}\right)$ the unique regular solution for the corresponding Tresca's problem and

$$
\begin{aligned}
& \tilde{\ell}_{m}\left(t, x^{\prime}\right)=\mathcal{F}\left(t, x^{\prime}, \sigma_{n}^{\ell_{m}}\right) \\
& =k(t) \int_{0}^{t} S(t-s)\left|\mathcal{R}\left(\sigma_{n}^{\ell_{m}}(\cdot, s)\right)\left(x^{\prime}\right)\right| d s \quad \forall x^{\prime} \in \Gamma_{0}, \forall t \in[0, T],
\end{aligned}
$$

with $\sigma^{\ell_{m}}=-p^{\ell_{m}} I+2 \mu(\theta) D\left(v^{\ell_{m}}+u_{0} \zeta\right)$.

Since $\tilde{\ell}_{m} \in W^{1, \infty}\left(0, T ; L_{+}^{2}\left(\Gamma_{0}\right)\right)$, we may assume without loss of generality (possibly modifying $\tilde{\ell}_{m}$ on a negligible subset of $\left.[0, T]\right)$ that $\tilde{\ell}_{m} \in C\left([0, T] ; L_{+}^{2}\left(\Gamma_{0}\right)\right)$ and with (44) and (48) we get

$$
\ell_{m+1}(t, \cdot)=\left\{\begin{array}{l}
\ell_{*}(t, \cdot) \text { on } \Gamma_{0} \times\left[0, \tau_{*}\right], \\
\ell_{*}\left(\tau_{*}, \cdot\right)+\int_{\tau_{*}}^{t} L_{m+1}(s, \cdot) d s=\ell_{*}\left(\tau_{*}, \cdot\right)+\int_{\tau_{*}}^{t} \frac{\partial \tilde{\ell}_{m}}{\partial s}(s, \cdot) d s \quad \text { on } \quad \Gamma_{0} \times\left[\tau_{*}, \tau\right], \\
\ell_{*}\left(\tau_{*}, \cdot\right)+\int_{\tau_{*}}^{\tau} L_{m+1}(s, \cdot) d s=\ell_{*}\left(\tau_{*}, \cdot\right)+\int_{\tau_{*}}^{\tau} \frac{\partial \tilde{\ell}_{m}}{\partial s}(s, \cdot) d s \quad \text { on } \quad \Gamma_{0} \times[\tau, T],
\end{array}\right.
$$

i.e.

$$
\ell_{m+1}(t, \cdot)=\ell_{*}\left(\tau_{*}, \cdot\right)+\left(\tilde{\ell}_{m}(t, \cdot)-\tilde{\ell}_{m}\left(\tau_{*}, \cdot\right)\right) \quad \text { on } \quad \Gamma_{0} \times\left[\tau_{*}, \tau\right]
$$

for all $m \geq 0$.

With the estimates obtained in Theorem 3.2, Proposition 3.3 and Proposition 3.4 we infer that $v^{\ell_{m}}, \frac{\partial^{2} v^{\ell_{m}}}{\partial t^{2}}, p^{\ell_{m}}$ and $\sigma^{\ell_{m}}$ are uniformly bounded in $W^{1, \infty}\left(0, T ; \mathbf{L}^{2}(\Omega)\right) \cap H^{1}\left(0, T ; \mathcal{V}_{0 d i v}\right)$, $L^{2}\left(0, T ;\left(\mathbf{H}_{0 d i v}^{1}(\Omega)\right)^{\prime}\right), L^{\infty}\left(0, T ; L_{0}^{2}(\Omega)\right), L^{\infty}(0, T ; F(\Omega))$ respectively and since $\Sigma$ maps $\mathcal{B}$ into itself we have also $L_{m}$ uniformly bounded in $L^{2}\left(\tau_{*}, \tau ; L^{2}\left(\Gamma_{0}\right)\right)$. 
Hence, possibly modifying $v^{\ell_{m}}$ and $\frac{\partial v^{\ell_{m}}}{\partial t}$ on a negligible subset of $[0, T]$, we have $v^{\ell_{m}} \in$ $C^{0}\left([0, T] ; \mathcal{V}_{0 d i v}\right)$ and $\frac{\partial v^{\ell_{m}}}{\partial t} \in C^{0}\left([0, T] ;\left(\mathbf{H}_{0 d i v}^{1}(\Omega)\right)^{\prime}\right)$. Moreover, possibly extracting a subsequence, we have

$$
\begin{gathered}
v^{\ell_{m}}, \frac{\partial v^{\ell_{m}}}{\partial t} \rightarrow v, \frac{\partial v}{\partial t} \quad \begin{array}{r}
\text { weakly star in } L^{\infty}\left(0, T ; \mathbf{L}^{2}(\Omega)\right) \\
\text { and weakly in } L^{2}\left(0, T ; \mathcal{V}_{0 d i v}\right),
\end{array} \\
\frac{\partial^{2} v^{\ell_{m}}}{\partial t^{2}} \rightarrow \frac{\partial^{2} v}{\partial t^{2}} \quad \text { weakly in } L^{2}\left(0, T ;\left(\mathbf{H}_{0 d i v}^{1}(\Omega)\right)^{\prime}\right), \\
p^{\ell_{m}} \rightarrow p \quad \text { weakly star in } L^{\infty}\left(0, T ; L_{0}^{2}(\Omega)\right), \\
L_{m} \rightarrow L \quad \text { weakly in } L^{2}\left(\tau_{*}, \tau ; L_{+}^{2}\left(\Gamma_{0}\right)\right),
\end{gathered}
$$

and, for all $t \in[0, T]$

$$
\begin{aligned}
& \ell_{m}(t, \cdot) \rightarrow \ell(t, \cdot)=\ell_{*}(t, \cdot) \quad \text { strongly in } L^{2}\left(\Gamma_{0}\right), \text { if } t \in\left[0, \tau_{*}\right], \\
& \ell_{m}(t, \cdot) \rightarrow \ell(t, \cdot)=\ell_{*}\left(\tau_{*}, \cdot\right)+\int_{\tau_{*}}^{t} L(s, \cdot) d s \quad \text { weakly in } L^{2}\left(\Gamma_{0}\right), \text { if } t \in\left[\tau_{*}, \tau\right], \\
& \ell_{m}(t, \cdot) \rightarrow \ell(t, \cdot)=\ell_{*}\left(\tau_{*}, \cdot\right)+\int_{\tau_{*}}^{\tau} L(s, \cdot) d s \quad \text { weakly in } L^{2}\left(\Gamma_{0}\right), \text { if } t \in[\tau, T] .
\end{aligned}
$$

By using Aubin's and Simon's lemmas, and possibly extracting another subsequence, we have also

$$
\frac{\partial v^{\ell_{m}}}{\partial t} \rightarrow \frac{\partial v}{\partial t} \quad \text { strongly in } L^{2}\left(0, T ; \mathbf{L}^{2}(\Omega)\right)
$$

and

$$
v^{\ell_{m}} \rightarrow v \quad \text { strongly in } C^{0}\left([0, T] ; \mathbf{L}^{4}(\Omega)\right)
$$

Moreover, by using again Aubin's lemma (see Theorem 2.1 page 271 in [30] with $X_{0}=\mathcal{V}_{0}$, $X_{1}=\mathbf{L}^{2}(\Omega)$ and $X=\mathbf{H}^{s}(\Omega)$ with $\left.\frac{1}{2}<s<1\right)$ we have also

$$
v^{\ell_{m}} \rightarrow v \quad \text { strongly in } L^{2}\left(0, T ; \mathbf{H}^{s}(\Omega)\right),
$$

and with the trace theorem we infer

$$
v^{\ell_{m}} \rightarrow v \quad \text { strongly in } L^{2}\left(0, T ; \mathbf{L}^{2}\left(\Gamma_{0}\right)\right) .
$$

Possibly modifying $v$ and $\frac{\partial v}{\partial t}$ on a negligible subset of $[0, T]$, we have $v \in C\left([0, T] ; \mathcal{V}_{0 d i v}\right)$ and $\frac{\partial v}{\partial t} \in C\left([0, T] ;\left(\mathbf{H}_{0 d i v}^{1}(\Omega)\right)^{\prime}\right)$. Similarly recalling that $f \in H^{1}\left(0, T ; \mathbf{L}^{2}(\Omega)\right)$, possibly modifying $f$ 
on a negligible subset of $[0, T]$, we have $f \in C\left([0, T] ; \mathbf{L}^{2}(\Omega)\right)$. By passing to the limit as $m$ tends to $+\infty$ in the corresponding Tresca's problem we obtain that $(v, p)$ is the unique regular solution on $[0, T]$ of Tresca's problem corresponding to a friction threshold given by $\ell$. Since $\ell \in H^{1}\left(0, T ; L_{+}^{2}\left(\Gamma_{0}\right)\right)$ we infer that $\sigma=\sigma^{\ell}=-p \operatorname{Id}+2 \mu(\theta) D\left(v+u_{0} \zeta\right)$ satisfies $\sigma \in L^{\infty}(0, T ; F(\Omega))$. With the previous convergence properties it is obvious that

$$
\sigma^{\ell_{m}} \rightarrow \sigma=\sigma^{\ell} \quad \text { weakly in } L^{2}\left(0, T ;\left(L^{2}(\Omega)\right)^{3 \times 3}\right)
$$

But, this weak convergence does not allow us to pass to the limit in the nonlinear relation (49). Nevertheless, by using compactness arguments based on Helly's theorem we may obtain:

Proposition 4.3. Let us assume that (10), (13)-(17) and (45) hold. Then

$$
\tilde{\ell}_{m}\left(t, x^{\prime}\right) \rightarrow \tilde{\ell}\left(t, x^{\prime}\right)=k(t) \int_{0}^{t} S(t-s)\left|\mathcal{R}\left(\sigma_{n}^{\ell}(s, \cdot)\right)\left(x^{\prime}\right)\right| d s \quad \forall x^{\prime} \in \Gamma_{0}, \forall t \in[0, T] .
$$

and

$$
\tilde{\ell}_{m} \rightarrow \tilde{\ell} \quad \text { strongly in } L^{2}\left((0, T) \times \Gamma_{0}\right)
$$

Proof. Since $\left(\frac{\partial v^{\ell_{m}}}{\partial t}\right)_{m \geq 0}$ converges strongly in $L^{2}\left(0, T ; \mathbf{L}^{2}(\Omega)\right)$ to $\frac{\partial v}{\partial t}$, we infer that

$$
\left\|\frac{\partial v^{\ell_{m}}}{\partial t}-\frac{\partial v}{\partial t}\right\|_{\mathbf{L}^{2}(\Omega)} \rightarrow 0 \quad \text { strongly in } L^{2}(0, T)
$$

and, possibly extracting a subsequence still denoted $\left(v^{\ell_{m}}, p^{\ell_{m}}\right)_{m \geq 0}$, there exists a negligible subset $A$ of $(0, T)$ such that

$$
\frac{\partial v^{\ell_{m}}}{\partial t}(t, \cdot) \rightarrow \frac{\partial v}{\partial t}(t, \cdot) \quad \text { strongly in } \mathbf{L}^{2}(\Omega) \text {, for all } t \in(0, T) \backslash A .
$$

On the other hand, the sequence $\left(v^{\ell_{m}}\right)_{m \geq 0}$ is bounded in $H^{1}\left(0, T ; \mathcal{V}_{0 \text { div }}\right) \subset H^{1}\left(0, T ; \mathbf{H}^{1}(\Omega)\right)$, so it is bounded in $C^{0}\left([0, T] ; \mathbf{H}^{1}(\Omega)\right)$ and, for all $t \in[0, T]$, there exists another subsequence of $\left(v^{\ell_{m}}\right)_{m \geq 0}$ such that $\left(v^{\ell_{m}}(t, \cdot)\right)_{m \geq 0}$ converges weakly in $\mathbf{H}^{1}(\Omega)$ but this subsequence depends on $t$. In order to avoid this difficulty we observe that $\left(v^{\ell_{m}}\right)_{m \geq 0}$ is bounded both in $C^{0}\left([0, T] ; \mathbf{H}^{1}(\Omega)\right)$ and in $B V\left(0, T ; \mathbf{H}^{1}(\Omega)\right)$. So we may apply compactness results for functions of bounded variations, namely Helly's theorem (see Theorem 2.1 page 10 in [22] for instance): from any sequence of functions from $[0, T]$ with values in a Hilbert space $X$ which is uniformly bounded in norm and in variation, we can extract a subsequence which converges pointwise weakly in $X$ to some function of $B V(0, T ; X)$. Thus we obtain that, possibly extracting another subsequence, still denoted $\left(v^{\ell_{m}}, p^{\ell_{m}}\right)_{m \geq 0}$, we have the following pointwise (this is the key point) convergence property:

$$
v^{\ell_{m}}(t, \cdot) \rightarrow \Lambda(t, \cdot) \quad \text { weakly in } \mathbf{H}^{1}(\Omega), \text { for all } t \in[0, T]
$$

with $\Lambda \in B V\left(0, T ; \mathbf{H}^{1}(\Omega)\right)$. Then, for all $\varphi \in \mathbf{H}^{1}(\Omega)$ and for all $\chi \in \mathcal{D}(0, T)$ we have

$$
\left(v^{\ell_{m}}(t, \cdot), \varphi\right)_{\mathbf{H}^{1}(\Omega)} \chi(t) \rightarrow \underset{20}{(\Lambda(t, \cdot), \varphi)_{\mathbf{H}^{1}(\Omega)}} \underset{ }{(t)} \text { for all } t \in[0, T]
$$


where $(\cdot, \cdot)_{\mathbf{H}^{1}(\Omega)}$ denotes the inner product of $\mathbf{H}^{1}(\Omega)$ and

$$
\left|\left(v^{\ell_{m}}(t, \cdot), \varphi\right)_{\mathbf{H}^{1}(\Omega)} \chi(t)\right| \leq\|\chi\|_{\mathcal{C}([0, T])}\|\varphi\|_{\mathbf{H}^{1}(\Omega)}\left\|v^{\ell_{m}}\right\|_{L^{\infty}\left(0, T ; \mathbf{H}^{1}(\Omega)\right)} \quad \text { for all } t \in[0, T] .
$$

We may apply Lebesgue's dominated convergence theorem and we get

$$
\int_{0}^{T}\left(v^{\ell_{m}}(t, \cdot), \varphi\right)_{\mathbf{H}^{1}(\Omega)} \chi(t) d t \rightarrow \int_{0}^{T}(\Lambda(t, \cdot), \varphi)_{\mathbf{H}^{1}(\Omega)} \chi(t) d t .
$$

Owing that $\left(v^{\ell_{m}}\right)_{m \geq 0}$ converges weakly to $v$ in $L^{2}\left(0, T ; \mathcal{V}_{0 \text { div }}\right)$, we infer that $\Lambda=v$ in $L^{2}\left(0, T ; \mathbf{H}^{1}(\Omega)\right)$, i.e.

$$
\int_{0}^{T}\|\Lambda(t, \cdot)-v(t, \cdot)\|_{\mathbf{H}^{1}(\Omega)}^{2} d t=0
$$

It follows that there exists another negligible subset $A^{\prime}$ of $(0, T)$ such that

$$
\Lambda(t, \cdot)=v(t, \cdot) \quad \text { in } \mathbf{H}^{1}(\Omega), \text { for all } t \in(0, T) \backslash A^{\prime}
$$

and with (53) we obtain

$$
v^{\ell_{m}}(t, \cdot) \rightarrow \Lambda(t, \cdot)=v(t, \cdot) \quad \text { weakly in } \mathbf{H}^{1}(\Omega), \text { for all } t \in(0, T) \backslash A^{\prime} .
$$

Recalling that, $\frac{\partial v^{\ell_{m}}}{\partial t}$ belongs to $C\left([0, T],\left(\mathbf{H}_{0 d i v}^{1}(\Omega)\right)^{\prime}\right) \cap L^{\infty}\left(0, T, \mathbf{L}^{2}(\Omega)\right)$ for all $m \geq 0$, we infer that $\frac{\partial v^{\ell_{m}}}{\partial t}$ is weakly continuous with values in $\mathbf{L}^{2}(\Omega)$ on $[0, T]$ and

$$
\left\|\frac{\partial v^{\ell_{m}}}{\partial t}(t, \cdot)\right\|_{\mathbf{L}^{2}(\Omega)} \leq\left\|\frac{\partial v^{\ell_{m}}}{\partial t}\right\|_{L^{\infty}\left(0, T ; \mathbf{L}^{2}(\Omega)\right)} \quad \text { for all } t \in[0, T]
$$

(see Lemma 1.4 page 263 in [30]). Now, let $m \geq 0$. For all $t \in[0, T]$ we define $f_{m}(t, \cdot) \in \mathbf{H}^{-1}(\Omega)$ by

$$
\begin{aligned}
& \left\langle f_{m}(t, \cdot), \varphi\right\rangle_{\mathbf{H}^{-1}(\Omega), \mathbf{H}_{0}^{1}(\Omega)}=-\left(\frac{\partial v^{\ell_{m}}}{\partial t}(t, \cdot)+u_{0} \zeta^{\prime}(t), \varphi\right)-a\left(\theta ; v^{\ell_{m}}(t, \cdot)+u_{0} \zeta(t), \varphi\right) \\
& -b\left(v^{\ell_{m}}(t, \cdot)+u_{0} \zeta(t), v^{\ell_{m}}(t, \cdot)+u_{0} \zeta(t), \varphi\right)+(f(t, \cdot), \varphi) \quad \forall \varphi \in \mathbf{H}_{0}^{1}(\Omega) .
\end{aligned}
$$

Then, consider now $\varphi \in \mathbf{H}_{0 \text { div }}^{1}(\Omega)$. With (22), we obtain that

$$
\int_{0}^{T}\left\langle f_{m}(t, \cdot), \varphi\right\rangle_{\mathbf{H}^{-1}(\Omega), \mathbf{H}_{0}^{1}(\Omega)} \chi(t) d t=0 \quad \forall \chi \in \mathcal{D}(0, T) .
$$

So

$$
\left\langle f_{m}(t, \cdot), \varphi\right\rangle_{\mathbf{H}^{-1}(\Omega), \mathbf{H}_{0}^{1}(\Omega)}=0 \quad \text { a.e. in }(0, T)
$$

and, using the continuity of the mapping $t \mapsto\left\langle f_{m}(t, \cdot), \varphi\right\rangle_{\mathbf{H}^{-1}(\Omega), \mathbf{H}_{0}^{1}(\Omega)}$ on $[0, T]$, we infer that the previous equality is valid for all $t \in[0, T]$. It follows that there exists a mapping $\widetilde{p}^{\ell_{m}}:[0, T] \rightarrow$ $L_{0}^{2}(\Omega)$ such that, for all $t \in[0, T]$

$$
\left\langle f_{m}(t, \cdot), \varphi\right\rangle_{\mathbf{H}^{-1}(\Omega), \mathbf{H}_{0}^{1}(\Omega)}=\left\langle\nabla \widetilde{p}^{\ell_{m}}(t, \cdot), \varphi\right\rangle_{\mathcal{D}^{\prime}(\Omega), \mathcal{D}(\Omega)} \quad \forall \varphi \in \mathcal{D}(\Omega) .
$$


But, for all $t \in[0, T]$, we have $\widetilde{p}^{\ell_{m}}(t, \cdot) \in L_{0}^{2}(\Omega)$ and thus

$$
\begin{aligned}
& \left\langle\nabla \widetilde{p}^{\ell_{m}}(t, \cdot), \varphi\right\rangle_{\mathcal{D}^{\prime}(\Omega), \mathcal{D}(\Omega)}=-\left\langle\widetilde{p}^{\ell_{m}}(t, \cdot), \operatorname{div}(\varphi)\right\rangle_{\mathcal{D}^{\prime}(\Omega), \mathcal{D}(\Omega)} \\
& =-\left(\widetilde{p}^{\ell_{m}}(t, \cdot), \operatorname{div}(\varphi)\right) \quad \forall \varphi \in \mathcal{D}(\Omega) .
\end{aligned}
$$

It follows that, for all $t \in[0, T]$,

$$
\begin{aligned}
& \left(\widetilde{p}^{\ell_{m}}(t, \cdot), \operatorname{div}(\varphi)\right)=\left(\frac{\partial v^{\ell_{m}}}{\partial t}(t, \cdot)+u_{0} \zeta^{\prime}(t), \varphi\right)+a\left(\theta ; v^{\ell_{m}}(t, \cdot)+u_{0} \zeta(t), \varphi\right) \\
& +b\left(v^{\ell_{m}}(t, \cdot)+u_{0} \zeta(t), v^{\ell_{m}}(t, \cdot)+u_{0} \zeta(t), \varphi\right)-(f(t, \cdot), \varphi) \quad \forall \varphi \in \mathcal{D}(\Omega)
\end{aligned}
$$

and by density of $\mathcal{D}(\Omega)$ into $\mathbf{H}_{0}^{1}(\Omega)$, the same equality is valid for all $\varphi \in \mathbf{H}_{0}^{1}(\Omega)$. With the same arguments as in Proposition 3.3, we obtain also that $\widetilde{p}^{\ell_{m}} \in L^{\infty}\left(0, T ; L_{0}^{2}(\Omega)\right)$ and $p^{\ell_{m}}=\widetilde{p}^{\ell_{m}}$ in $L^{\infty}\left(0, T ; L_{0}^{2}(\Omega)\right)$. Thus possibly modifying $p^{\ell_{m}}$ on a negligible subset of $(0, T)$ we have

$$
\begin{aligned}
& \left(p^{\ell_{m}}(t, \cdot), \operatorname{div}(\varphi)\right)=\left(\frac{\partial v^{\ell_{m}}}{\partial t}(t, \cdot)+u_{0} \zeta^{\prime}(t), \varphi\right)+a\left(\theta ; v^{\ell_{m}}(t, \cdot)+u_{0} \zeta(t), \varphi\right) \\
& +b\left(v^{\ell_{m}}(t, \cdot)+u_{0} \zeta(t), v^{\ell_{m}}(t, \cdot)+u_{0} \zeta(t), \varphi\right)-(f(t, \cdot), \varphi) \quad \forall \varphi \in \mathbf{H}_{0}^{1}(\Omega), \quad \forall t \in[0, T] .
\end{aligned}
$$

Similarly, possibly modifying $p$ on a negligible subset of $(0, T)$, we have

$$
\begin{aligned}
& (p(t, \cdot), \operatorname{div}(\varphi))=\left(\frac{\partial v}{\partial t}(t, \cdot)+u_{0} \zeta^{\prime}(t), \varphi\right)+a\left(\theta ; v(t, \cdot)+u_{0} \zeta(t), \varphi\right) \\
& +b\left(v(t, \cdot)+u_{0} \zeta(t), v(t, \cdot)+u_{0} \zeta(t), \varphi\right)-(f(t, \cdot), \varphi) \quad \forall \varphi \in \mathbf{H}_{0}^{1}(\Omega), \quad \forall t \in[0, T] .
\end{aligned}
$$

Now let $\tilde{w} \in L^{2}(\Omega)$ and $w \in L_{0}^{2}(\Omega)$ be given by

$$
w=\tilde{w}-\frac{1}{|\Omega|} \int_{\Omega} \tilde{w} d x
$$

For all $m \geq 0$ and for all $t \in[0, T]$ we have

$$
\begin{aligned}
& \left(p^{\ell_{m}}(t, \cdot)-p(t, \cdot), \tilde{w}\right)=\left(p^{\ell_{m}}(t, \cdot)-p(t, \cdot), w\right) \\
& =\left(\frac{\partial v^{\ell_{m}}}{\partial t}(t, \cdot)-\frac{\partial v}{\partial t}(t, \cdot), P(w)\right)+a\left(\theta ; v^{\ell_{m}}(t, \cdot)-v(t, \cdot), P(w)\right) \\
& +b\left(v^{\ell_{m}}(t, \cdot)-v(t, \cdot), v^{\ell_{m}}(t, \cdot)+u_{0} \zeta(t), P(w)\right)+b\left(v(t, \cdot)+u_{0} \zeta(t), v^{\ell_{m}}(t, \cdot)-v(t, \cdot), P(w)\right)
\end{aligned}
$$

where $P$ is the linear continuous operator from $L_{0}^{2}(\Omega)$ into $\mathbf{H}_{0}^{1}(\Omega)$ such that $\operatorname{div}(P(w))=w$ for all $w \in L_{0}^{2}(\Omega)$. With (51), (52) and (55) we get

$$
\left(p^{\ell_{m}}(t, \cdot)-p(t, \cdot), \tilde{w}\right) \rightarrow 0 \quad \text { for all } \tilde{w} \in L^{2}(\Omega), \text { for all } t \in(0, T) \backslash\left(A \cup A^{\prime}\right) .
$$

By using the definition of $\mathcal{F}$ we obtain

$$
\begin{aligned}
& \left|\mathcal{F}\left(t, x^{\prime}, \sigma_{n}^{\ell_{m}}\right)-\mathcal{F}\left(t, x^{\prime}, \sigma_{n}^{\ell}\right)\right|=k(t)\left|\int_{0}^{t} S(t-s)\left(\left|\mathcal{R}\left(\sigma_{n}^{\ell_{m}}(s, \cdot)\right)\left(x^{\prime}\right)\right|-\left|\mathcal{R}\left(\sigma_{n}^{\ell}(s, \cdot)\right)\left(x^{\prime}\right)\right|\right) d s\right| \\
& \leq k(t) \int_{0}^{t} S(t-s)\left|\mathcal{R}\left(\sigma_{n}^{\ell_{m}}(s, \cdot)-\sigma_{n}^{\ell}(s, \cdot)\right)\left(x^{\prime}\right)\right| d s \quad \forall x^{\prime} \in \Gamma_{0}, \forall t \in[0, T] .
\end{aligned}
$$


Thus, with the definition of $\mathcal{R}$ and Stokes formula (40), we have

$$
\begin{aligned}
& \left|\mathcal{F}\left(t, x^{\prime}, \sigma_{n}^{\ell_{m}}\right)-\mathcal{F}\left(t, x^{\prime}, \sigma_{n}^{\ell}\right)\right| \\
& \leq k(t) \int_{0}^{t} S(t-s)\left|\left(\sigma^{\ell_{m}}(s, \cdot)-\sigma^{\ell}(s, \cdot), \operatorname{grad} \tilde{\varphi}_{x^{\prime}}\right)_{\left(L^{2}(\Omega)\right)^{3 \times 3}}\right| d s \\
& +k(t) \int_{0}^{t} S(t-s)\left|\left\langle\operatorname{div}\left(\sigma^{\ell_{m}}(s, \cdot)-\sigma^{\ell}(s, \cdot)\right), \tilde{\varphi}_{x^{\prime}}\right\rangle_{\mathbf{L}^{4 / 3}(\Omega), \mathbf{L}^{4}(\Omega)}\right| d s
\end{aligned}
$$

and with Remark 3.2

$$
\begin{aligned}
& \left|\mathcal{F}\left(t, x^{\prime}, \sigma_{n}^{\ell_{m}}\right)-\mathcal{F}\left(t, x^{\prime}, \sigma_{n}^{\ell}\right)\right| \\
& \leq\|k\|_{L^{\infty}(0, T)}\|S\|_{C([0, T])} \int_{0}^{t}\left(\left|\left(p(s, \cdot)-p^{\ell_{m}}(s, \cdot), \operatorname{div}\left(\tilde{\varphi}_{x^{\prime}}\right)\right)\right|+\left|a\left(\theta ; v^{\ell_{m}}(s, \cdot)-v(s, \cdot), \tilde{\varphi}_{x^{\prime}}\right)\right|\right) d s \\
& +\|k\|_{L^{\infty}(0, T)}\|S\|_{\mathcal{C}([0, T])} \int_{0}^{t}\left|\left(\frac{\partial v^{\ell_{m}}}{\partial t}(s, \cdot)-\frac{\partial v}{\partial t}(s, \cdot), \tilde{\varphi}_{x^{\prime}}\right)\right| d s \\
& +\|k\|_{L^{\infty}(0, T)}\|S\|_{\mathcal{C}([0, T])} \int_{0}^{t}\left|b\left(v^{\ell_{m}}(s, \cdot)-v(s, \cdot), v^{\ell_{m}}(s, \cdot)+u_{0} \zeta(s), \tilde{\varphi}_{x^{\prime}}\right)\right| d s \\
& +\|k\|_{L^{\infty}(0, T)}\|S\|_{\mathcal{C}([0, T])} \int_{0}^{t}\left|b\left(v(s, \cdot)+u_{0} \zeta(s), v^{\ell_{m}}(s, \cdot)-v(s, \cdot), \tilde{\varphi}_{x^{\prime}}\right)\right| d s \quad \forall x^{\prime} \in \Gamma_{0}, \forall t \in[0, T] .
\end{aligned}
$$

With the previous convergences results (51), (52), (55) and (58), we infer that, for all $x^{\prime} \in \Gamma_{0}$ :

$$
\begin{gathered}
\left(p(s, \cdot)-p^{\ell_{m}}(s, \cdot), \operatorname{div}\left(\tilde{\varphi}_{x^{\prime}}\right)\right) \rightarrow 0 \quad \forall s \in[0, T] \backslash\left(A \cup A^{\prime}\right), \\
a\left(\theta ; v^{\ell_{m}}(s, \cdot)-v(s, \cdot), \tilde{\varphi}_{x^{\prime}}\right) \rightarrow 0 \quad \forall s \in[0, T] \backslash A^{\prime}, \\
\left(\frac{\partial v^{\ell_{m}}}{\partial t}(s, \cdot)-\frac{\partial v}{\partial t}(s, \cdot), \tilde{\varphi}_{x^{\prime}}\right) \rightarrow 0 \quad \forall s \in[0, T] \backslash A, \\
b\left(v^{\ell_{m}}(s, \cdot)-v(s, \cdot), v^{\ell_{m}}(s, \cdot)+u_{0} \zeta(s), \tilde{\varphi}_{x^{\prime}}\right) \rightarrow 0 \quad \forall s \in[0, T] \backslash A^{\prime},
\end{gathered}
$$

and

$$
b\left(v(s, \cdot)+u_{0} \zeta(s), v^{\ell_{m}}(s, \cdot)-v(s, \cdot), \tilde{\varphi}_{x^{\prime}}\right) \rightarrow 0 \quad \forall s \in[0, T] \backslash A^{\prime} .
$$

Owing that $v^{\ell_{m}}, \frac{v^{\ell_{m}}}{\partial t}$ and $p^{\ell_{m}}$ are uniformly bounded in $L^{\infty}\left(0, T ; \mathbf{H}^{1}(\Omega)\right), L^{\infty}\left(0, T ; \mathbf{L}^{2}(\Omega)\right)$ and $L^{\infty}\left(0, T ; L_{0}^{2}(\Omega)\right)$ respectively, we may apply Lebesgue's dominated convergence theorem and we conclude that

$$
\mathcal{F}\left(t, x^{\prime}, \sigma_{n}^{\ell_{m}}\right) \rightarrow \mathcal{F}\left(t, x^{\prime}, \sigma_{n}^{\ell}\right) \quad \forall x^{\prime} \in \Gamma_{0}, \forall t \in[0, T]
$$

Moreover, recalling that $\mathcal{R} \in \mathcal{L}_{c}\left(F(\Omega) ; C\left(\Gamma_{0}\right)\right)$, we get

$$
\begin{aligned}
& \left|\mathcal{F}\left(t, x^{\prime}, \sigma_{n}^{\ell_{m}}\right)\right| \\
& \leq T\|k\|_{L^{\infty}(0, T)}\|S\|_{\mathcal{C}([0, T])}\|\mathcal{R}\|_{\mathcal{L}_{c}\left(F(\Omega) ; C\left(\Gamma_{0}\right)\right)}\left\|\sigma^{\ell_{m}}\right\|_{L^{\infty}(0, T ; F(\Omega))} \quad \forall x^{\prime} \in \Gamma_{0}, \forall t \in[0, T]
\end{aligned}
$$


and with Proposition 3.4

$$
\begin{aligned}
& \left|\mathcal{F}\left(t, x^{\prime}, \sigma_{n}^{\ell_{m}}\right)\right| \\
& \leq T\|k\|_{L^{\infty}(0, T)}\|S\|_{C([0, T])}\|\mathcal{R}\|_{\mathcal{L}_{c}\left(F(\Omega) ; C\left(\Gamma_{0}\right)\right)} C_{\text {data }}\left(1+\left\|\frac{\partial \ell_{m}}{\partial t}\right\|_{L^{2}\left(0, T ; L^{2}\left(\Gamma_{0}\right)\right)}\right) \\
& \leq T\|k\|_{L^{\infty}(0, T)}\|S\|_{\mathcal{C}([0, T])}\|\mathcal{R}\|_{\mathcal{L}_{c}\left(F(\Omega) ; C\left(\Gamma_{0}\right)\right)} C_{\text {data }} \\
& \times\left(1+\left(\left\|\frac{\partial \ell_{*}}{\partial t}\right\|_{L^{2}\left(0, \tau_{*} ; L^{2}\left(\Gamma_{0}\right)\right)}^{2}+\left\|L_{m}\right\|_{L^{2}\left(\tau_{*}, \tau ; L^{2}\left(\Gamma_{0}\right)\right)}^{2}\right)^{1 / 2}\right) \\
& \leq T\|k\|_{L^{\infty}(0, T)}\|S\|_{\mathcal{C}([0, T])}\|\mathcal{R}\|_{\mathcal{L}_{c}\left(F(\Omega) ; C\left(\Gamma_{0}\right)\right)} C_{\text {data }} \\
& \times\left(1+\frac{\sqrt{\alpha} \exp \left(-\mathbf{A}_{3} T\right)}{\sqrt{7} \gamma(\Omega)}\left(\frac{\alpha^{3}}{9 K^{4}}-\mathcal{D}_{0}^{\prime}\right)^{1 / 2}\right) \quad \forall x^{\prime} \in \Gamma_{0}, \forall t \in[0, T] .
\end{aligned}
$$

Hence, by using again Lebesgue's dominated convergence theorem, we infer

$$
\tilde{\ell}_{m}=\mathcal{F}\left(\cdot, \cdot, \sigma_{n}^{\ell_{m}}\right) \rightarrow \mathcal{F}\left(\cdot, \cdot, \sigma_{n}^{\ell}\right) \quad \text { strongly in } L^{2}\left((0, T) \times \Gamma_{0}\right) .
$$

Let us consider now $\tau_{*}=0$ and $\ell_{*} \equiv 0$. Then there exists $\rho>0$, depending only on the data, such that for any $\tau \in(0, \rho)$, all the previous results hold. In particular, $\ell_{m+1}(t, \cdot)=\tilde{\ell}_{m}(t, \cdot)$ for all $m \geq 0$ and for all $t \in(0, \tau)$, which yields at the limit $\ell=\tilde{\ell}$. Hence $(v, p)$ is a solution of Tresca's problem (22)-(24) with a shear stress threshold $\ell=\mathcal{F}\left(\cdot, \cdot, \sigma_{n}\right)$ i.e. $(v, p)$ is a solution of problem (19)-(21) on $[0, \tau]$ and we may conclude

Theorem 4.4. (Existence for Coulomb's problem in the 3D case) Let us assume that (10) and (13)-(17) hold. Assume moreover that

$$
\mathcal{D}_{0}^{\prime}=\mathcal{D}_{0}+\left(\mathbf{A}_{\mathbf{0}}^{\mathbf{2}}+2\left\|\frac{\partial f}{\partial t}\right\|_{L^{2}\left(0, T ; \mathbf{L}^{2}(\Omega)\right)}^{2}+2 \mathbf{A}_{\mathbf{1}} T+2 \mathbf{A}_{2} C_{1}^{2}\right) \exp \left(2 \mathbf{A}_{\mathbf{3}} T\right)<\frac{\alpha^{3}}{9 K^{4}}
$$

where $\mathcal{D}_{0}$ and $\mathbf{A}_{\mathbf{i}}$ for $i \in\{0, \ldots, 3\}$ are defined as in Section 3. Then there exists $\rho>0$, depending only on the data, such that for any $\tau \in(0, \rho)$, problem $(P)$ admits at least a solution $(v, p)$ on $[0, \tau]$ satisfying $v \in W^{1, \infty}\left(0, \tau ; \mathbf{L}^{2}(\Omega)\right) \cap H^{1}\left(0, \tau ; \mathcal{V}_{0 \text { div }}\right), \frac{\partial^{2} v}{\partial t^{2}} \in L^{2}\left(0, \tau ;\left(\mathbf{H}_{0 \text { div }}^{1}(\Omega)\right)^{\prime}\right), p \in$ $L^{\infty}\left(0, \tau ; L_{0}^{2}(\Omega)\right), \sigma=-p I+2 \mu(\theta) D\left(v+u_{0} \zeta\right) \in L^{\infty}(0, \tau ; F(\Omega))$ and $\mathcal{F}\left(\cdot, \cdot, \sigma_{n}\right) \in W^{1, \infty}\left(0, \tau ; L^{2}\left(\Gamma_{0}\right)\right)$. Moreover, if

$$
\begin{aligned}
& \frac{7 \gamma(\Omega)^{2}}{\alpha}\left(C_{\text {data }}^{\prime} C_{\text {data }}\right)^{2}\left(1+\frac{\sqrt{\alpha} \exp \left(-\mathbf{A}_{\mathbf{3}} T\right)}{\sqrt{7} \gamma(\Omega)}\left(\frac{\alpha^{3}}{9 K^{4}}-\mathcal{D}_{0}^{\prime}\right)^{1 / 2}\right)^{2} T \exp \left(2 \mathbf{A}_{\mathbf{3}} T\right) \\
& \leq \frac{\alpha^{3}}{9 K^{4}}-\mathcal{D}_{0}^{\prime}
\end{aligned}
$$

then $\rho=T$ and we get a solution of problem $(P)$ on $[0, T]$. 
Remark 4.1. Let us observe that we do not obtain here a solution $(v, p)$ to Coulomb's friction problem (19)-(21) by using a fixed point theorem with respect to $\left(L_{m}\right)_{m \geq 0}$ but by using a successive approximation technique with respect to the shear stress threshold i.e. by studying a limit process for the sequence shear stress thresholds $\left(\ell_{m}\right)_{m \geq 0}$ and the corresponding solutions $\left(v^{\ell_{m}}, p^{\ell_{m}}\right)_{m \geq 0}$ of Tresca's problem (22)-(24).

Remark 4.2. Let us consider the $2 D$ case and assume that (10) and (13)-(17) hold. Then condition (26) can be avoided and, for any $\ell \in H^{1}\left(0, T ; L_{+}^{2}\left(\Gamma_{0}\right)\right)$, Tresca's problem admits a unique solution $(v, p)$ such that $v \in W^{1, \infty}\left(0, T ; \mathbf{L}^{2}(\Omega)\right) \cap H^{1}\left(0, T ; \mathcal{V}_{0 \text { div }}\right)$ and $p \in L^{2}\left(0, T ; L_{0}^{2}(\Omega)\right.$ ) (see [2]). Thus with the same computations as in Proposition 3.3 and Proposition 3.4 we may still prove that

$$
\frac{\partial^{2} v}{\partial t^{2}} \in L^{2}\left(0, T ;\left(\mathbf{H}_{0 d i v}^{1}(\Omega)\right)^{\prime}\right), \quad p \in L^{\infty}\left(0, T ; L_{0}^{2}(\Omega)\right)
$$

and

$$
\sigma=-p I+2 \mu(\theta) D\left(v+u_{0} \zeta\right) \in L^{\infty}(0, T ; F(\Omega)) .
$$

But without condition (26) we loose the uniform estimate (29). Nevertheless by using the classical Sobolev's inequality

$$
\|u\|_{\mathbf{L}^{4}(\Omega)} \leq C(\Omega)\|u\|_{\mathbf{L}^{2}(\Omega)}^{1 / 2}\|u\|_{\mathbf{H}^{1}(\Omega)}^{1 / 2} \quad \forall u \in \mathbf{H}^{1}(\Omega)
$$

we get

$$
\begin{aligned}
& \left|\left\langle b\left(v+u_{0} \zeta, v+u_{0} \zeta, \varphi\right), \chi\right\rangle\right| \\
& \leq C(\Omega)\left\|v+u_{0} \zeta\right\|_{L^{\infty}\left(0, T ; \mathbf{L}^{2}(\Omega)\right)}^{1 / 2}\left\|v+u_{0} \zeta\right\|_{L^{\infty}\left(0, T ; \mathbf{H}^{1}(\Omega)\right)}\left\|v+u_{0} \zeta\right\|_{L^{2}\left(0, T ; \mathbf{H}^{1}(\Omega)\right)}^{1 / 2}\|\varphi \chi\|_{L^{4 / 3}\left(0, T ; \mathbf{L}^{4}(\Omega)\right)}
\end{aligned}
$$

for all $\varphi \in \mathcal{V}_{0}$ and for all $\chi \in \mathcal{D}(0, T)$. Moreover (35) is replaced by (see Proposition 4.1 in [2])

$$
\begin{aligned}
& \left\|\frac{\partial v_{\varepsilon m}^{\delta}}{\partial t}(s)\right\|_{\mathbf{L}^{2}(\Omega)}^{2}+\frac{2}{\delta} \int_{0}^{s}\left\|\operatorname{div}\left(\frac{\partial v_{\varepsilon m}^{\delta}}{\partial t}\right)\right\|_{L^{2}(\Omega)}^{2} d t+\alpha \int_{0}^{s}\left\|\frac{\partial v_{\varepsilon m}^{\delta}}{\partial t}\right\|_{\mathbf{H}^{1}(\Omega)}^{2} d t \\
& \leq \mathbf{A}_{\mathbf{4}} \exp \left(2 \mathbf{A}_{\mathbf{3}} s+\frac{3^{7} 7^{3}}{2^{8} \alpha^{4}} C(\Omega)^{8}\left(C_{1}^{\prime}\right)^{2} \exp \left(2 C_{1}^{\prime \prime} s\right)\right) \quad \forall s \in[0, T] .
\end{aligned}
$$

Hence estimate (37) is replaced by

$$
\|\sigma\|_{L^{4}(0, T ; F(\Omega))} \leq C_{\text {data }}\left(1+\left\|\frac{\partial \ell}{\partial t}\right\|_{L^{2}\left(0, T ; L^{2}\left(\Gamma_{0}\right)\right)}\right)
$$

with a positive constant $C_{\text {data }}$ depending only on the data $\mu, \zeta, f, u_{0}$ and $\theta$. By a straightforward adaptation of Proposition 4.2 we get $\mathcal{F}\left(\cdot, \cdot, \sigma_{n}\right) \in W^{1,4}\left(0, T ; L^{2}\left(\Gamma_{0}\right)\right)$ with

$$
\left\|\mathcal{F}\left(\cdot, \cdot, \sigma_{n}\right)\right\|_{W^{1,4}\left(0, T ; L^{2}\left(\Gamma_{0}\right)\right)} \leq C_{\text {data }}^{\prime}\|\sigma\|_{L^{4}(0, T ; F(\Omega))}
$$

with a positive constant $C_{\text {data }}^{\prime}$ depending only on $\|\mathcal{R}\|_{\mathcal{L}_{c}\left(F(\Omega) ; C\left(\Gamma_{0}\right)\right)}$ and on the data $S$ and $k$. 
Then we may construct in the same way the mapping $\Sigma$ and, using the same notations as previously,

$$
\begin{aligned}
& \|\Sigma(L)\|_{L^{2}\left(\tau_{*}, \tau ; L^{2}\left(\Gamma_{0}\right)\right)} \leq\left(\tau-\tau_{*}\right)^{1 / 4}\|\Sigma(L)\|_{L^{4}\left(\tau_{*}, \tau ; L^{2}\left(\Gamma_{0}\right)\right)} \leq\left(\tau-\tau_{*}\right)^{1 / 4}\|\tilde{\ell}\|_{W^{1,4}\left(0, T ; L^{2}\left(\Gamma_{0}\right)\right)} \\
& \leq C_{\text {data }}^{\prime} C_{\text {data }}\left(\tau-\tau_{*}\right)^{1 / 4}\left(1+\left\|\frac{\partial \ell}{\partial t}\right\|_{L^{2}\left(0, T ; L^{2}\left(\Gamma_{0}\right)\right)}\right) \\
& \leq C_{\text {data }}^{\prime} C_{\text {data }}\left(\tau-\tau_{*}\right)^{1 / 4}\left(1+\left(\left\|\frac{\partial \ell_{*}}{\partial t}\right\|_{L^{2}\left(0, \tau_{*} ; L^{2}\left(\Gamma_{0}\right)\right)}^{2}+\|L\|_{L^{2}\left(\tau_{*}, \tau ; L^{2}\left(\Gamma_{0}\right)\right)}^{2}\right)^{1 / 2}\right) \\
& \leq C_{\text {data }}^{\prime} C_{\text {data }}\left(\tau-\tau_{*}\right)^{1 / 4}\left(1+\left\|\frac{\partial \ell_{*}}{\partial t}\right\|_{L^{2}\left(0, \tau_{*}: L^{2}\left(\Gamma_{0}\right)\right)}+\|L\|_{L^{2}\left(\tau_{*}, \tau ; L^{2}\left(\Gamma_{0}\right)\right)}\right)
\end{aligned}
$$

Let $\rho \in\left(0, \frac{1}{\left(C_{\text {data }}^{\prime} C_{\text {data }}\right)^{4}}\right)$. Then for any $\tau \in\left(\tau_{*}, \tau_{*}+\rho\right)$ and for any $R>0$ such that

$$
\frac{C_{\text {data }}^{\prime} C_{\text {data }}\left(\tau-\tau_{*}\right)^{1 / 4}}{1-C_{\text {data }}^{\prime} C_{\text {data }}\left(\tau-\tau_{*}\right)^{1 / 4}}\left(1+\left\|\frac{\partial \ell_{*}}{\partial t}\right\|_{L^{2}\left(0, \tau_{*}: L^{2}\left(\Gamma_{0}\right)\right)}\right)<R
$$

$\Sigma$ maps the ball $\bar{B}_{L^{2}\left(\tau_{*}, \tau ; L^{2}\left(\Gamma_{0}\right)\right)}(0, R)$ into itself and we may apply the successive approximation technique described previouly, leading first to a solution $(v, p)$ of problem $(P)$ on $[0, \tau]$ for any $\tau \in(0, \rho)$. By choosing then $\tau_{*} \in(0, \rho)$ and $\ell_{*}$ as the restriction of $\mathcal{F}\left(\cdot, \cdot, \sigma_{n}\right)$ to $\left[0, \tau_{*}\right]$, with $\sigma=p I+2 \mu(\theta) D\left(v+u_{0} \zeta\right)$, we can build with the same technique, for any $\tau \in\left(\tau_{*}, \tau_{*}+\rho\right)$, an extension of $(v, p)$ to $[0, \tau]$ which is a solution of problem $(P)$ on $[0, \tau]$. Since $\rho$ is independent of $\tau_{*}$ and $\ell_{*}$ we may conclude with a finite induction that

Theorem 4.5. (Existence for Coulomb's problem in the 2D case) Let us assume that (10) and (13)-(17) hold. Then problem $(P)$ admits at least a solution $(v, p)$ on $[0, T]$ satisfying $v \in$ $W^{1, \infty}\left(0, T ; \mathbf{L}^{2}(\Omega)\right) \cap H^{1}\left(0, T ; \mathcal{V}_{0 d i v}\right), \frac{\partial^{2} v}{\partial t^{2}} \in L^{2}\left(0, T ;\left(\mathbf{H}_{0 d i v}^{1}(\Omega)\right)^{\prime}\right), p \in L^{\infty}\left(0, T ; L_{0}^{2}(\Omega)\right), \sigma \in$ $L^{\infty}(0, T ; F(\Omega))$ and $\mathcal{F}\left(\cdot, \cdot, \sigma_{n}\right) \in W^{1, \infty}\left(0, T ; L^{2}\left(\Gamma_{0}\right)\right)$.

\section{References}

[1] M. Boukrouche, R. El Mir. On the Navier-Stokes system in a thin film flow with Tresca free boundary condition and its asymptotic behavior, Bull. Math. Soc. Sc. Math. Roumanie 48 (96-2) (2005) 139-163.

[2] M. Boukrouche, I. Boussetouan, L. Paoli. Non-isotermal Navier-Stokes system with mixed boundary conditions and friction law: uniqueness and regularity properties, Nonlinear Anal. TMA, 102 (2014) 168-185

[3] M. Boukrouche, I. Boussetouan, L. Paoli. Existence for non-isothermal fluid flows with Tresca's friction and Cattaneo's heat law, J. Math. Anal. Appl., 427 (2015) 499-514.

[4] M. Boukrouche, I. Boussetounn, L. Paoli. Unsteady 3D-Navier-Stokes system with Tresca's friction law, arXiv:1512.06607 [math.AP] (2015).

[5] M. Boukrouche, L. Paoli. Global existence for a 3D non-stationary Stokes flow with Coulomb's type friction boundary conditions, ArXiv:1607.01592 [math.AP] (2016).

[6] M. Boukrouche, F. SAIDI. Non isothermal lubrication problem with Tresca fluid-solid interface law, part I, Nonlinear Anal. Real World App., 7(5) (2006) 1145-1166.

[7] C.A. Coulomb. Théorie des machines simples, Bachelier, Paris, 1821.

[8] L. Demkowicz, J.T. Oden. On some existence and uniqueness results in contact problems with nonlocal friction, Nonlinear Analysis TMA, 6(10) (1982) 1075-1093.

[9] G. Duvaut, J.L. Lions. Les inéquations en mécanique et physique. Dunod, Gauthiers-Villars, Paris, 1972.

[10] G. Duvaut. Équilibre d'un solide élastique avec contact unilatéral et frottement de Coulomb, C.R. Acad. Sci. Paris, 290 (1980) 263-265. 
[11] G. Duvaut. Loi de frottement non locale, Journal de Mécanique Théorique et Appliquée, Numéro spécial (1982) 73-78.

[12] H. Fujita. Flow problems with unilateral boundary conditions, Leçons au Collège de France (1993).

[13] H. Fustra. A mathematical analysis of motions of viscous incompressible fluid under leak or slip boundary conditions, Math. Fluid Mech. Model., 888 (1994) 199-216.

[14] H. Fujta. Non-stationary Stokes flows under leak boundary conditions of friction type, J. Comput. Appl. Math., 19 (2001) 1-8.

[15] H. Fusta. A coherent analysis of Stokes flows under boundary conditions of friction type, J. Comput. Appl. Math., 149(1) (2002) 57-69.

[16] V. Girault, P.A. Raviart. Finite element approximation of the Navier-Stokes equations, Springer-Verlag, Berlin, 1979.

[17] H. Hervet, L. LÉGER. Flow with slip at the wall: from simple to complex fluids, C. R. Acad. Sci. Paris, Physique 4 (2003) 241-249.

[18] I.R. Ionescu, M. Sofonea. The blocking property in the study of Bingham fluids, Int. J. Engng. Sci., 24(3) (1986) 289-297.

[19] V.A. Kondrat'ev, O.A. Oleinik. Boundary-value problems for the system of elasticity theory in unbounded domains. Korn's inequalities, Russian Math. Surveys 43(5) (1988) 65-119.

[20] J.L. Lions. Some problems connected with Navier-Stokes equations, Lectures at the $I V^{T H}$ Latin-American School of Mathematics, Lima, July 1978.

[21] A. Magnin, J.M. Piau. Shear rheometry of fluids with a yield stress, J. Non-Newtonian Fluid Mech., 23 (1987) 91-106.

[22] M. Monteiro Marques. Differential inclusions in non-smooth mechanical problems: shocks and dry friction. Birkhauser, Boston, Berlin, 1993.

[23] P.A. Raviart, J.M. Thomas. Introduction à l'analyse numérique des équations aux dérivées partielles. Masson, Paris, 1988.

[24] I.J. Rao, K.R. Rajagopal. The effect of the slip boundary condition on the flow of fluids in a channel, Acta Mechanica, 135 (1999) 113-126.

[25] R.T. Rockafellar. Convex analysis, Princeton University Press, Princeton, 1970.

[26] T. Roubicek. Nonlinear partial differential equations with applications, Birkhaüser Verlag, Basel, 2005.

[27] N. Saito, H. Fujita. Regularity of solutions to the Stokes equation under a certain nonlinear boundary condition, The Navier-Stokes equations, Lecture Note Pure Appl. Math., 223 (2001) 73-86.

[28] N. Saito. On the Stokes equations with the leak and slip boundary conditions of friction type: regularity of solutions, Publ. RIMS Kyoto Univ., 40 (2004) 345-383.

[29] M. Sofonea, A. Farcas. Analysis of a history-dependent frictional contact problem, Appl. Anal., 93(2) (2014) 428-444.

[30] R. Temam. Navier-Stokes equations, North-Holland, Amsterdam, New-York, Oxford, 1984. 